\title{
Einblicke in die Gründe für nicht-normalverteilte Immobilienrenditen: eine explorative Untersuchung deutscher Wohnimmobilienportfolios
}

\author{
Patrick Krieger $(\mathbb{D}) \cdot$ Carsten Lausberg • Kristin Wellner
}

Eingegangen: 26. Juni 2017 / Überarbeitet: 7. April 2019 / Angenommen: 14. Juni 2019 / Online publiziert: 22. Juli 2019

(C) Der/die Autor(en) 2019

Zusammenfassung Dass die Renditen von direkten Immobilieninvestments nicht normalverteilt sind, gilt als erwiesen. Aber warum Immobilienrenditen nicht normalverteilt sind, wurde bisher kaum erforscht. Das ist erstaunlich, denn die Normalverteilung wird in Wissenschaft und Praxis immer noch häufig unterstellt, d.h. man verwendet ein systematisch fehlerhaftes Modell ohne Erkenntnisse über Ursachen und Wirkungen des Fehlers zu haben, um dem Fehler so Rechnung tragen zu können. Im Risikomanagement, wo viele Risikomaße auf Verteilungen basieren, muss das zu Fehlentscheidungen führen.

Wir gehen davon aus, dass die Erforschung der Gründe für nicht-normalverteilte Immobilienrenditen zur Verfeinerung von Risikomodellen und zur Verbesserung des Immobilienrisikomanagements beitragen kann. Daher ist unser Ziel, ausgewählte Gründe in einem explorativen Verfahren zu untersuchen. Durch unsere Stichprobe sind die Ergebnisse zwar auf Direktinvestitionen in deutsche Wohnraumportfolios beschränkt, aber gewisse Aspekte sind vermutlich auf gewerbliche und ausländische Immobilien sowie indirekte Investitionen übertragbar. Insgesamt legen die Ergebnisse nahe, dass (a) die Theorie deterministisch-chaotischer Prozesse ungeeignet für Direktinvestments ist, (b) Immobilienzeitreihen durch deterministische Ereignisse geprägt sind, die eine Messung verzerren und (c) sich das Risiko mehrdimensional durch die Variablen Homogenität, Granularität und Frequenz ergibt. Auch wenn

\footnotetext{
P. Krieger $(\triangle)$

Technische Universität Berlin, Kaiser-Friedrich-Straße 39, 10117 Berlin, Deutschland

E-Mail: patrick.krieger@campus.tu-berlin.de

C. Lausberg

Immobilienwirtschaftliches Institut für IT, Hochschule für Wirtschaft und Umwelt

Nürtingen-Geislingen, Parkstraße 4, 73312 Geislingen/Steige, Deutschland

K. Wellner

Fakultät VI Bauen Planen Umwelt, Technische Universität Berlin, Straße des 17.

Juni 152, 10623 Berlin, Deutschland
} 
diese Ergebnisse sehr vorsichtig interpretiert werden sollten, geben die Ergebnisse Anlass zu einer neuen, tiefergehenden Diskussion der bisherigen Risikomessmethoden.

Schlüsselwörter Direktinvestment · Risikomessung · Nicht-Normalverteilung ·

Deterministisches Chaos $\cdot$ Sprungprozesse

\title{
Insights into the reasons for non-normal real estate returns: An explorative study of German housing portfolios
}

\begin{abstract}
It can be considered as mere fact that the returns on direct real estate investments are not normally distributed. But why real estate returns are not normally distributed has hardly been researched. That is astonishing because the normal distribution is still frequently assumed in academia and practice, i.e., a systematically flawed model is used without knowledge about the causes and effects of the error, which would be necessary to account for the error. In risk management, where many risk measures are based on distributions, this must lead to wrong decisions.

We assume that research into the reasons for non-normally distributed real estate returns can contribute to refining risk models and improving real estate risk management. Therefore, our goal is to examine selected reasons in an exploratory approach. Even though the results of our sample are limited to direct investments in German housing portfolios, certain aspects are probably transferable to commercial and foreign properties as well as indirect investments. Overall, the results suggest that (a) the theory of deterministic-chaotic processes is inappropriate for direct investments, (b) real estate time series are characterised by deterministic events that distort a measurement, and (c) the risk is multi-dimensional, driven by the variables homogeneity, granularity and frequency. Even though the results should be interpreted very carefully, they give rise for new profound discussions of current risk measurement practices.
\end{abstract}

Keywords Direct investment · Risk measurement · Non-normal returns · Deterministic-chaos $\cdot$ Jump processes

\section{Einführung in die Immobilienrisikoforschung und Vorstellung der Forschungsmethode}

Zahlreiche Studien sind in den letzten Jahren zu dem Ergebnis gekommen, dass Immobilienrenditen nicht normalverteilt sind. „Na und?“ könnte man fragen, schließlich ist die Normalverteilung nur ein Spezialfall unter den Häufigkeitsverteilungen, und die Annahme, dass sich die Renditen von so etwas Komplexem wie Immobilieninvestitionen durch so etwas Regelmäßiges wie die Normalverteilung darstellen lassen, ist dem realitätsfernen Modell der Portfoliotheorie geschuldet. Dennoch ist das Ergebnis mindestens aus den folgenden drei Gründen bemerkenswert: Erstens macht es darauf aufmerksam, dass noch nicht klar ist, wie die Verteilung der Renditen wirklich aussieht und welche Faktoren sie bestimmen. Zweitens zerstört das 


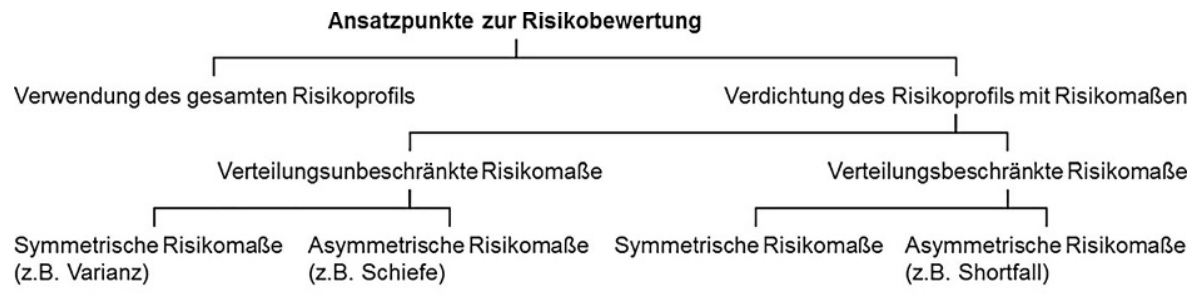

Abb. 1 Übersicht zu Ansatzpunkten in der Risikobewertung (Eigene Darstellung in Anlehnung an Baumeister (2004), S. 17)

Ergebnis manche liebgewonnene Angewohnheit, z. B. die Volatilität unreflektiert als Risikokennzahl zu verwenden. Drittens fordert es zum Nachdenken darüber auf, nach welchen Kriterien Entscheidungen über Immobilieninvestitionen getroffen werden sollten. Wir widmen uns in diesem Artikel vor allem dem ersten Punkt und stellen daraufhin Überlegungen zu den anderen beiden Punkten an. Bevor wir unsere Herangehensweise erläutern, wollen wir kurz den Wissensstand zur Verteilung von Immobilienrenditen skizzieren.

Die erwartete Rendite ist für einen professionellen Investor das wichtigste Kriterium bei der Bewertung einer Investition (French 2001). Sie drückt den natürlichen Wunsch des Investors nach einer Belohnung für das eingegangene Risiko und die aufgegebene Liquidität aus. Die Rendite ist für einen Investor der nächstliegende Eckpunkt des Kapitalanlagedreiecks ${ }^{1}$, während die anderen beiden Eckpunkte ihm weniger vertraut sind (Strümpel 1968, S. 95 ff.; Luhmann 1997, S. 327 ff.), er wenig Gespür für sie hat (Gigerenzer 2013; Kahneman 2016) und deren Kennzahlen weniger erforscht sind. Die Liquidität kann auch als Teilrisiko betrachtet werden, so dass die Betrachtung der Einfachheit halber auf Rendite und Risiko reduziert wird (Pástor und Stambaugh 2003, S. 644). Das Risiko schließlich wird häufig aus der Verteilung der Rendite abgeleitet, vor allem aus den Streuungsmaßen Varianz, Standardabweichung und Volatilität.

Praxis und Wissenschaft haben zur Messung des Risikos viele Kennzahlen entwickelt, für die zum Beispiel Baumeister (2004, S. 17) eine übersichtliche, wenn auch unvollständige, weil auf quantitative Größen beschränkte Einteilung bereitstellt (Abb. 1).

Grundsätzlich kann man zur Risikobewertung die vollständige Verteilung berücksichtigen oder das Risiko mittels Kennzahlen verdichten. Die vollständige (empirische) Verteilung ist für Immobilien aus Datenmangel meist nicht bekannt. Daher wird auf idealisierte Modelle zurückgegriffen, wie zum Beispiel die Normalverteilung, die durch die erwartete Rendite und die Standardabweichung vollständig beschrieben wird (zurückgehend auf Markowitz 1952, und von Neumann und Morgenstern 1944). Des Weiteren können Risikomaße danach unterschieden werden, ob nur ein Teil der Verteilung zur Berechnung des Risikos betrachtet werden sollte

\footnotetext{
${ }^{1}$ Als sog. ,magisches Dreieck der Kapitalanlage“ bezeichnet man das Konkurrenzverhältnis, in dem die wichtigsten Kapitalanlageziele Rentabilität, Sicherheit und Liquidität zueinanderstehen. Neuere Ansätze erweitern das Dreieck um weitere Ziele, zum Beispiel Ethik oder Nachhaltigkeit, und den Faktor Zeit.
} 
(ursprünglicher Gedanke von Roy 1952); Kennzahlen wie die Semi-Volatilität beschränken sich auf den Bereich unterhalb der erwarteten Rendite. Dadurch nimmt die Zahl der notwendigen Kennzahlen zu, was eine Entscheidung erschwert. Um die steigende Komplexität durch weitere Kennzahlen zu reduzieren, wurden risikoadjustierte Performancemaße entwickelt. Hierzu gehört bspw. der Risk-adjusted Return on Capital (RAROC), der ursprünglich für Banken entwickelt wurde (Schierenbeck et al. 2008), aber mittlerweile auch Anwendung in Immobiliengesellschaften gefunden hat. Dennoch werden quantitative Risikomaße in der Immobilienwirtschaft eher stiefmütterlich behandelt, es dominieren qualitative Methoden wie die Szenarioanalyse (Plantz 2012, S. 120). Die Gründe sind nicht genau erforscht, aber es gibt Hinweise, dass es Praktikern schwerfällt, Risikomaße mit typischen Maßnahmen der immobilienwirtschaftlichen Praxis zu verbinden (Pfnür und Armonat 2001).

Den quantitativen Risikomaßen ist gemein, dass ihnen zur Berechnung oftmals ein Verteilungsmodell zugrunde liegt. Es können auch verteilungsfreie Methoden eingesetzt werden, die aber eine geringere Teststärke besitzen und mit einer höheren Komplexität des Modells einhergehen. Daher erfreut sich die Normalverteilung als Verteilungsmodell immer noch großer Beliebtheit. Dies wäre nicht problematisch, wenn das Modell in Bezug auf die empirischen Phänomene bei Immobiliendaten hinreichend präzise wäre - ist es aber nicht, wie u. a. die Untersuchungen von Müller et al. (2014), Young (2008), Young et al. (2006), Wellner (2003), Lizieri und Ward (2001) sowie Young und Graff (1995) belegen. Die Abweichungen vom Modell zeigen sich vor allem in den spitzeren Wölbungen (Leptokurtosis) und stärkeren Enden (Fat-Tails) von Immobilienrenditen. ${ }^{2}$

Es gibt nach unserer Ansicht vier grundsätzliche Strategien, mit dem Problem der Nicht-Normalverteilung umzugehen. Die erste Strategie ist das Ignorieren einer Normal-Verteilung, die natürlich nur sinnvoll ist, wenn davon ausgegangen werden kann, dass dadurch kein systematischer Fehler entsteht. Angesichts der deutlichen Abweichungen in den bisherigen Untersuchungen, insbesondere am linken Rand der Verteilung, ist dies nicht empfehlenswert (s. Taleb 2007). Des Weiteren ist der Forschungsstand über Verteilungen immobilienwirtschaftlicher Größen zu gering, um von einer Unbedenklichkeit ausgehen zu können. Eine weitere Strategie ist die in der Statistik übliche Transformation der Eingangsvariablen. Hier gibt es diverse Verfahren, um spezielle Charakteristika von Querschnitts- und Längsschnittdaten zu korrigieren, z.B. die Trendbereinigung, das Logarithmieren nicht-linearer Daten oder die Transformation nach Box und Cox (1964). Leider ist dies nicht immer wirkungsvoll und mündet trotz aller Bemühungen oft in fehlerbehaften oder instabilen Modellen. Die dritte Strategie ist die Verwendung von Verfahren, die robust gegen Ausreißer und asymmetrische Verteilungen sind. Eine Möglichkeit sind verteilungsfreie Modelle wie beispielsweise die Nutzung des Medians, der Mean Absolute Deviation oder Shrinkage-Verfahren. Letzteres soll die Weiterverwendung der Normalverteilung und den Erhalt ihrer Vorzüge ermöglichen, wie es beispielsweise der Ansatz von Ledoit und Wolf (2003) zeigt. Die letzte Strategie versucht Verteilungs-

\footnotetext{
2 Nicht-Normalität kommt in unterschiedlichen Ausprägungen vor. Neben der von der Normalverteilung abweichenden Form der Verteilung sind z. B. Autokorrelation und Heteroskedastizität zu nennen, auf die aber hier nicht weiter eingegangen wird.
} 


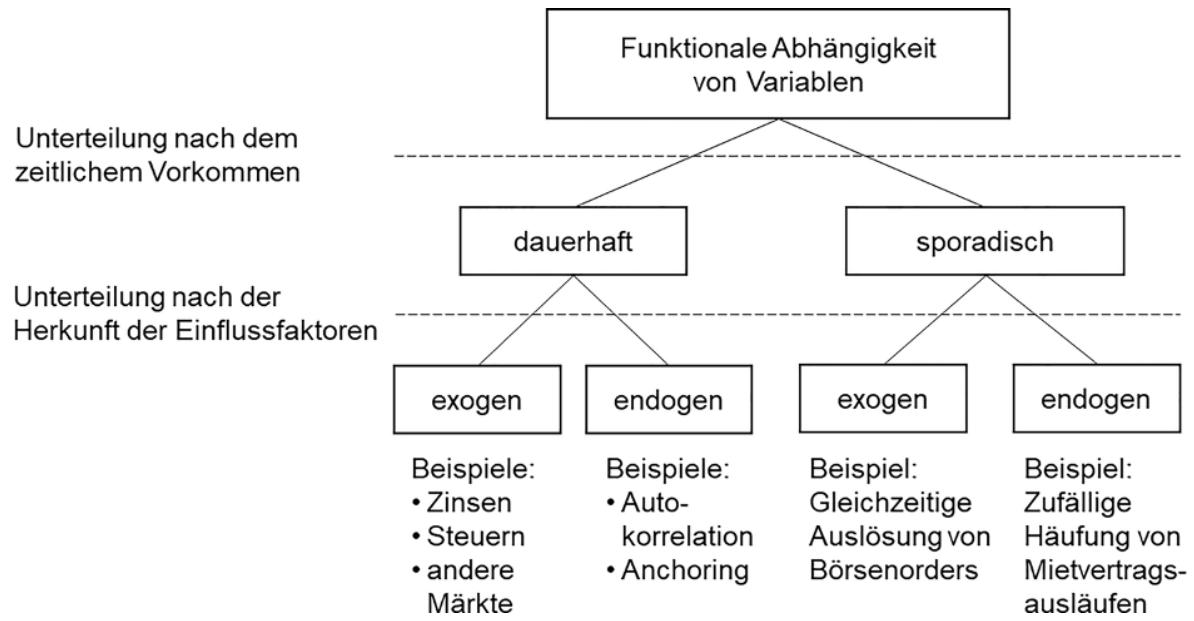

Abb. 2 Unterteilung von Abhängigkeiten zweier oder mehrerer Variablen

modelle zu nutzen, die eine bessere Anpassung an die Charakteristika empirischer Daten bieten. Beispiele hierfür sind die relativ gut erforschte t-Verteilung (Bollerslev 1987), Sprungverteilungen (siehe bspw. Cont und Tankov 2004) oder auch die sehr flexible alpha-stabile Verteilung (Mandelbrot 1960). Zum einen gewähren solche Modelle eine präzisere Anpassung an empirische Daten ${ }^{3}$, zum anderen immunisieren sie die Messung statistischer Parameter gegen Ausreißer. Dieser Ansatz kann jedoch akzeptierte ökonomische Axiome verletzen und in mathematisch komplexe Lösungen münden.

Alle vier Strategien sind prinzipiell nützlich, doch ignorieren sie die Gründe für die Nicht-Normalverteilung von Renditen. Die wahren Gründe sind leider bisher kaum bekannt, es existieren derzeit lediglich Vermutungen und abstrakte mathematische Beschreibungen (Cont 2001), die wenig Praxisbezug aufweisen. Daher soll dies der erste Aufsatz sein, der das Phänomen der Nicht-Normalität versucht zu ergründen, anstatt es zu umgehen, zu beschreiben oder zu modellieren.

Die folgenden Ausführungen versuchen die bisherigen Erkenntnisse zu kategorisieren. Nach unserer Einschätzung gelingt das am besten nach dem Kriterium der funktionalen Abhängigkeit der Variablen. ${ }^{4}$

Die erste Kategorie der Gründe für nicht-normale Renditeverteilungen bilden dauerhafte Abhängigkeiten, die am besten durch ein Korrelationsmaß beschrieben werden können - siehe Abb. 2. Hier kann man weiter in Abhängigkeiten von exogenen und von endogenen Faktoren unterteilen. Die Forschung konzentriert sich überwie-

\footnotetext{
3 Siehe hierzu die Literaturanalyse in Abschnitt „Die Messung von Veränderungen in Immobilienmärkten und -portfolios".

4 Die kausale Abhängigkeit ist zwar ein Teil der funktionalen Abhängigkeit, steht hierbei aber nicht im Mittelpunkt. Große Änderungen können auch durch zeitliche Abfolgen entstehen, was unter dem Aspekt der Causa (siehe „cum hoc ergo propter hoc“) uninteressant, jedoch für die Nicht-Normalverteilung eine Bedeutung hat.
} 
gend auf exogene Faktoren wie Zinsen, Inflation und Einkommen (siehe z. B. Lieser und Groh 2014 und Kurzrock et al. 2009). Auch Steueranreize haben über ihren Einfluss auf Bautätigkeit und Mietentwicklung (Smith et al. 2000; Gallagher und Wood 1999) einen permanenten Bezug zu Immobilienrenditen. Dass „dauerhaft““ nicht „konstant“ bedeutet, sieht man unter anderem daran, dass die Korrelationen zwischen Finanzmärkten in Wirtschaftskrisen ansteigen und so die Diversifikation eines Portfolios verringern (Sandoval Jr. und Franca 2012). Die Korrelationen zwischen Immobilienmärkten sind ebenfalls instabil (Schindler 2009; Lee 2006), was ein wesentlicher Grund für Abweichungen von der Normalverteilung ist.

Die Autokorrelation, also die Abhängigkeit des aktuellen Wertes von Werten aus vorherigen Perioden, ist eine endogene Korrelation, die ebenfalls als Grund für Nicht-Normalverteilung angeführt wird (Mandelbrot 1960). Die Gründe für ihre Existenz sind nicht restlos geklärt, obwohl es sich um einen der größten Streitpunkte in der Diskussion um das Konzept der Markteffizienz handelt (Fama 1970, 1965; Mandelbrot 1963). Die Autokorrelation tritt insbesondere bei Immobilienbewertungen auf, da eine aktuelle Bewertung zum Teil auf den Informationen früherer Gutachten beruht (Brown und Matysiak 2000). In Immobilienmärkten wie auch in anderen Märkten mit ausgeprägten Zyklen wurden schon häufig Autokorrelationen nachgewiesen (Wheaton 1987; Liow 2007; Grenadier 1995). Außerdem existieren psychologische Faktoren, die zu den endogenen Gründen gezählt werden können. Northcraft und Neale (1987) haben zum Beispiel nachgewiesen, dass Immobiliengutachter an vielen verschiedenen Werten ,,ankern“ (wie ihren eigenen, früher ermittelten Werten (Diaz und Wolverton 1998)) und somit Immobilien systematisch über- oder unterbewerten. Tritt die Ernüchterung im Markt ein, kommt es zu einer Marktkorrektur bei Transaktionspreisen. Diese Marktkorrekturen fallen zudem noch stärker aus als rational angebracht wäre (DeBondt und Thaler 1986), was Ausreißer begünstigt.

Die zweite Kategorie bilden sporadische Abhängigkeiten, die naturgemäß nur selten zu beobachten sind, im Immobilienkontext wenig erforscht wurden und schwierig zu modellieren sind. Ein gutes Beispiel dafür sind exogene Schocks, wie die Änderung von Steuergesetzen. Das Gewähren und spätere Auslaufen von Steuervorteilen für Immobilien hat direkten, aber zeitlich begrenzten Einfluss auf die Renditen. ${ }^{5}$ Der Transmissionsmechanismus ist dabei oft kompliziert, wie bei dem bekannten Beispiel des Börsenkrachs vom 19. Oktober 1987: Die Ergebnisse von Shiller (1987) legen nahe, dass es zu einer zufälligen Angleichung der Handelsstrategien kam, die zu den massiven Kurseinbrüchen geführt hat. Ein weiteres Beispiel sind die Mietvertragsrestlaufzeiten beim liquidierten Immobilienfonds Hansaimmobilia (Hansainvest 2011). Ein Drittel aller Mietverträge lief ohne erkennbaren Grund innerhalb von nur einer Periode aus.

\footnotetext{
5 Indirekt führen derartige Änderungen nicht selten zu Immobilienbooms und -krisen, was die Bedeutung sporadischer Abhängigkeiten unterstreicht. Beispiele sind die „Sonderabschreibung Ost“ in Deutschland in den 1990er Jahren (Henger und Voigtländer, 2015) oder die durch das Economic Recovery Tax Act 1981 in den USA eingeführten und mit dem Tax Reform Act 1986 wieder abgeschafften Steuervorteile (Lausberg 2001, S. 117).
} 
Zusammengefasst kann man sagen, dass Abhängigkeiten zu Ausreißern und einer stärkeren Wölbung im Vergleich zur Normalverteilung führen. Wie häufig sporadische im Vergleich zu dauerhaften Abhängigkeiten sind, wurde noch nicht untersucht. Mit Taleb (2007) lässt sich aber feststellen, dass höchst unwahrscheinliche Ereignisse einen großen Einfluss auf den Lauf der Geschichte haben - und auf Immobilienrenditeverteilungen ebenso!

Ziel dieses Artikels ist, die Gründe für diese Nicht-Normalverteilung explorativ zu untersuchen, wofür wir das Prinzip der Triangulation ${ }^{6}$ einsetzen (Yin 2014, S. $119 \mathrm{ff}$.). Die Untersuchung ist dabei auf Direktinvestments in deutsche Wohnimmobilienportfolios beschränkt. Die Abweichungen von der Normalverteilung werden aus drei verschiedenen Perspektiven analysiert, bei denen verschiedene Datensätze sowie quantitative und qualitative Methoden eingesetzt werden, um ein vollständigeres Bild zu erzeugen (Hammersley 2008). Die erste Perspektive konzentriert sich auf die Literatur, die alternative Modelle auf Basis unterschiedlicher Datensätze untersucht hat. Hierzu wurde eine umfangreiche Literaturrecherche zur Messung des Renditerisikos in Immobilienmärkten durchgeführt. Die zweite Perspektive fokussiert auf Beispiele, die zu Abweichungen von der Normalverteilung führen. Als erster Schritt werden die zuvor analysierten Modelle mit Renditedaten auf Objektebene angepasst, um die Genauigkeit der Modelle zu bestimmen. Für den zweiten Schritt konnten drei umfangreiche Datensätze mit Mieteinnahmen auf Portfolio-, Objekt- und Mietvertragsebene sowie Ausgaben auf Portfolioebene analysiert werden. Diese Datensätze sollen Hinweise auf die Gründe für Abweichungen von der Normalverteilung geben. Die dritte Perspektive untersucht die Wahrnehmung des Risikos durch die Beteiligten, da der Begriff „Risiko“ uneinheitlich definiert ist. (French 2001) Beim Risikobegriff handelt sich um ein soziales Konstrukt, das durch Subjektivität geprägt ist und ohne den Menschen als Entscheider nicht auskommt (Slovic 1999). Aus diesem Grund wird die Analyse durch drei Interviews ergänzt, die mit Experten aus dem Assetmanagement, dem Risikomanagement und der Bewertung geführt wurden.

\section{Die verwendeten Daten}

Es ist praktisch unmöglich, in einem Modell alle Entscheidungen zu berücksichtigen, die zu Änderungen in einem Immobilienportfolio führen können. Daher erfolgte eine Auswahl benötigter Daten auf Basis der Bestandteile des Total Returns, der auch den Einstiegspunkt in die Untersuchung von Risikomodellen bildet. Hierzu wurden uns von einem Assetmanager die Total Returns eines Immobilienportfolios zur

\footnotetext{
6 Triangulation hat zum Ziel, durch (mindestens drei) verschiedene Perspektiven eine höhere Validität zu erreichen, da sich die Fehler der jeweiligen Perspektiven gegenseitig aufheben (Denzin 1970, S. 297 ff.). Diese Annahme ist nicht ohne Kritik (Flick 1992; McFee 1992; Blaikie 1991). Als Konsens lässt sich feststellen, dass Triangulation zu einem reichhaltigeren, aber nicht unbedingt valideren Bild des Forschungsgegenstands führt (Hammersley 2008), was jedoch bewusst für diese Untersuchung genutzt wird.
} 
Verfügung gestellt (Zeitreihe A). ${ }^{7}$ Dieser institutionelle Investor verfügt über einen in Jahrzehnten aufgebauten Immobilienbestand überwiegend in deutschen A- und B-Städten. Das hier betrachtete Teilportfolio umfasst insgesamt 70 in Deutschland belegene Wohnimmobilien und gemischt genutzte Immobilien mit überwiegender Wohnnutzung. Die Total Returns erstrecken sich über einen Zeitraum von 2008 bis 2012. Zu Vergleichszwecken wurde der German Property Index Wohnen von BulwienGesa in die Untersuchung einbezogen (Zeitreihe B1). Er wurde ausgewählt, da er zum einen als reiner Wohn-Performanceindex publiziert wird und zum anderen eine hohe Datenqualität besitzt. Zum Vergleich wurden ferner die Kurse einer zufällig ausgewählten illiquiden Immobilienaktie herangezogen (Zeitreihe B2). Die für viele Immobilienaktien typische geringe Liquidität schlägt sich stark in der Häufigkeitsverteilung der Aktienrenditen nieder, weil die selten vorkommenden Transaktionen starke Kursausschläge bewirken.

Damit auch Eingangsvariablen des Total Returns untersucht werden, wurden weitere Daten in die Analyse einbezogen. Für die Cashflow-Untersuchungen wurden Daten von drei Immobilienportfolios verwendet, die uns von den jeweiligen Assetmanagern überlassen wurden. ${ }^{8}$ Die Portfolios $\mathrm{C} 1$ und $\mathrm{C} 2$ befinden sich jeweils in einer deutschen A-Stadt. Das Portfolio C1 umfasst insgesamt $92.317 \mathrm{~m}^{2}$ vermietbare Fläche, das Portfolio C2 $186.299 \mathrm{~m}^{2}$, in beiden Fällen vereinzelt auch Gewerbeflächen. Die Portfolios blieben während des Betrachtungszeitraums in ihrer vertraglichen Mietfläche und der Anzahl der Objekte unverändert und umfassen einen Zeitraum von Januar 2009 bis Dezember 2013. Die Daten der beiden Portfolios stehen lediglich auf Portfolioebene zur Verfügung, umfassen aber die Variablen SollMieteinnahmen ${ }^{9}(\mathrm{M})$, Leerstand (L), geplante Instandhaltung (gI), ungeplante Instandhaltung (uI) und Modernisierung (Mo). Das Portfolio C3 befindet sich in einer deutschen B-Stadt und bestand zum Juli 2014 aus einer vermietbaren Fläche von insgesamt $786.273 \mathrm{~m}^{2}$ in 13.008 Wohnungen, die sich auf 497 Wirtschaftseinheiten ${ }^{10}$ verteilen. Das Wohnportfolio wurde über den Betrachtungszeitraum von Januar 2011 bis Juli 2014 um 415 Wohnungen (ca. 3,1\%) erweitert, was einem Anstieg der vermietbaren Fläche von ca. 3,7\% entspricht. Dies ist der bisher detaillierteste Datensatz, der für eine derartige Untersuchung herangezogen wurde, er umfasst jedoch lediglich die Soll-Mieteinnahmen, keine Kostenpositionen. Tab. 1 gibt eine Übersicht über die verwendeten Daten.

Die empirische Literatur zu Risikomodellen zeigt, dass Renditen nur mit verschiedenartig strukturierten, stochastischen Signalen beschrieben werden können. (Assaf 2015) Diese stochastischen Modelle liefern aber keine Erklärung bezüglich

\footnotetext{
${ }^{7}$ Der Total Return wurde mit der Gleichung $r=\left(V_{-} t+C F_{-} t\right) / V_{-}(t-1)-1$ ermittelt, wobei $V_{-} t$ für den Marktwert in Periode $t$ steht, $C F_{-} t$ für den Cashflow der Periode $t$ und $V_{-}(t-1)$ für den Marktwert am Ende der Vorperiode.

8 Aus Gründen der Vertraulichkeit können keine Angaben zu Unternehmen, Orten u.ä. veröffentlicht werden.

9 Die Soll-Miete ist für die folgenden Ausführungen die am Monatsanfang buchhalterisch ins Soll gestellte Miete, die durch Zahlungseingang ausgeglichen wird. Es existieren in der Praxis auch alternative Definitionen, die aber hier aufgrund der Datenlage ungeeignet sind.

10 Wirtschaftseinheiten können aus einzelnen oder mehreren Gebäuden bestehen, die aus Gründen der Bewirtschaftung zusammengefasst werden.
} 
Tab. 1 Daten zur Ermittlung der Modellanpassung

\begin{tabular}{lllllll}
\hline Zeitreihe & Detailgrad & Kennzahl & Region & Zeitraum & Wohnfläche $^{\mathrm{a}}$ & Frequenz \\
\hline A & Portfolio & Total Return & DE & $2008-2012$ & - & Jährlich \\
B1 & Index & Total Return & DE & $1991-2017$ & - & Jährlich \\
B2 & Aktie & Kursrendite & DE & $03.2008-12.2012$ & - & Täglich \\
C1 & Portfolio & M-L-gI-uI-Mo DE & $01.2009-12.2013$ & $186.299 \mathrm{~m}^{2}$ & Monatlich \\
C2 & Portfolio & M-L-gI-uI-Mo DE & $01.2009-12.2013$ & $92.317 \mathrm{~m}^{2}$ & Monatlich \\
C3 & Wohnung & M-L & DE & $01.2011-07.2014$ & $786.273 \mathrm{~m}^{2}$ & Monatlich \\
\hline
\end{tabular}

${ }^{a}$ Hierbei handelt es sich um die im Mietvertrag vereinbarte Wohnfläche der Wohnung

der Leptokurtosis und den Fat tails. Des Weiteren muss berücksichtigt werden, dass sich Aggregatvariablen wie der Total Return aus mehreren Komponenten zusammensetzen, die wiederum eigene stochastische Merkmale aufweisen. Insbesondere der im Total Return enthaltene Marktwert integriert mit dem Werturteil des Gutachters die Risikoperspektive eines Dritten und kann als Unsicherheit zweiter Ordnung bezeichnet werden. Eine Berechnung des Total Returns auf Transaktionsbasis würde dieses Problem zwar umgehen, ist aber wiederum auf Portfolio- und Objekteebene für die Risikomessung nicht praktikabel, da ansonsten zu wenig Daten zur Verfügung stehen würden.

Der erste Schritt der Untersuchung soll klären, ob für die verschiedenen Zeitreihen eine Normalverteilung vorliegt. Die Forschungsfrage lautet:

F1: „Liegt eine Normalverteilung für die einzelnen Zeitreihen vor?““

Tab. 2 gibt eine Übersicht über die Daten und deren Analyse, die in der Untersuchung verwendet werden. Die Immobilienportfolios C1 bis C3 wurden für die allgemeine statistische Auswertung zusammengefasst, außer für die Variable Leerstand, da diese unterschiedlich berechnet wird. Der Leerstand von Portfolio C1 und Portfolio C2 wird in der Soll-Miete des Vormonats ausgedrückt, wohingegen der Leerstand von Portfolio C3 zu Marktwerten berechnet wird. Für alle Zeitreihen der drei Portfolios wurden die ersten Differenzen berechnet, um eine stationäre Zeitreihe herzustellen. Für die Zeitreihen A, B1 und B2 wurden die logarithmierten Renditen berechnet, um die Stetigkeit der Variablen (im statistischen Sinne) zu gewährleisten. Bis auf die Variable Leerstand weichen alle Zeitreihen von der Normalverteilung auf einem Signifikanzniveau von unter $1 \%$ ab. ${ }^{11}$ Dies ist ein häufiges Merkmal empirischer Zeitreihen, aber die hohe Exzess-Kurtosis der Soll-Miete ist bemerkenswert. Auch der Kurs der Immobilienaktie weist eine hohe Kurtosis auf, die in Verbindung mit der Illiquidität der Aktie bzw. der geringen Granularität der Nachfrage gebracht wird (Cristelli et al. 2010).

Zur Komplettierung des Datenbestands wurden zudem Presseberichte und unstrukturierte Experteninterviews verwendet, um Ähnlichkeiten und Strukturen von Sprüngen aufzudecken. Die drei Interviewpartner sind branchenbekannte Fachleute mit langer Berufserfahrung im ausgeübten Tätigkeitsfeld (Assetmanagement, Ri-

11 Es wurden sowohl der Andersen-Darling-Test (A-D) als auch der Jarque-Bera-Test (J-B) Test verwendet. 
Tab. 2 Übersichtsstatistiken zu den standardisierten Zeitreihen

\begin{tabular}{|c|c|c|c|c|c|c|c|c|}
\hline Zeitreihe & $N$ & StdAbw & Schiefe & Kurtosis & $\begin{array}{l}A-D- \\
\text { Test }\end{array}$ & $p$ & $J-B-T e s t$ & $P$ \\
\hline$\Delta$ Soll-Miete & 160 & 0,2779 & 11,4862 & 140,2635 & 42,8184 & $* * *$ & $126.543,70$ & $* * *$ \\
\hline$\Delta$ Leerstand & 118 & 0,0180 & 0,1009 & $-0,3556$ & 0,4164 & - & 0,95 & - \\
\hline $\begin{array}{l}\Delta \text { Geplante In- } \\
\text { standhaltung }\end{array}$ & 118 & 0,8094 & 1,1953 & 13,2049 & 11,0760 & $* * *$ & 808,15 & $* * *$ \\
\hline $\begin{array}{l}\Delta \text { Ungeplante } \\
\text { Inst }\end{array}$ & 118 & 0,2112 & 0,2130 & 3,1411 & 2,2580 & $* * *$ & 43,92 & $* * *$ \\
\hline $\begin{array}{l}\Delta \text { Modernisie- } \\
\text { rung }\end{array}$ & 118 & 1,0112 & 1,0912 & 6,5482 & 4,0755 & $* * *$ & 213,26 & $* * *$ \\
\hline $\begin{array}{l}\operatorname{Ln}(1+\text { Total Re- } \\
\text { turn A) }\end{array}$ & 279 & 0,0773 & 3,0241 & 17,0336 & 19,9460 & $* * *$ & 2759,6000 & $* * *$ \\
\hline $\begin{array}{l}\text { Ln }(1+\text { Total Re- } \\
\text { turn B1) }\end{array}$ & 27 & 0,0901 & 1,9998 & 5,6747 & 1,2634 & $* * *$ & 73,4590 & $* * *$ \\
\hline Ln(Aktie B2) & 773 & 0,1194 & 2,8590 & 44,4072 & 168,6200 & $* * *$ & $64.924,0000$ & $* * *$ \\
\hline
\end{tabular}

$* * *$ entspricht Signifikanzniveau $p \leq 1 \%$, ** entspricht $p \leq 5 \%$, * entspricht $p \leq 10 \%$

sikomanagement, Bewertung), die in drei bekannten Immobilienunternehmen in Deutschland arbeiten.

\section{Die Messung von Veränderungen in Immobilienmärkten und -portfolios}

Wie oben beschrieben, legen die empirischen Befunde nahe, dass die Normalverteilungsannahme für die Risikomessung ungeeignet ist. Durch sie entsteht ein hohes Modellrisiko, da die empirischen Verteilungen ökonomischer Variablen von einer Normalverteilung gravierend abweichen, insbesondere wird die Häufigkeit sehr großer Abweichungen unterschätzt. Allerdings ist die Normalverteilung wegen ihrer Einfachheit immer noch sehr beliebt. Außerdem herrscht Uneinigkeit darüber, welche alternativen Modelle geeignet sind. Aus dieser andauernden Diskussion haben sich zwei unterschiedliche Lösungen entwickelt: (1) ein theoretischer Ansatz deterministisch-chaotischer Prozesse und (2) ein pragmatischer Ansatz sprungartiger Prozesse. Die erste Perspektive der Triangulation soll mittels einer ausführlichen Literaturrecherche prüfen, ob ein dominantes und hinreichend präzises Modell existiert. Die diametral gelagerten Forschungsfragen hierfür lauten:

F2a: „Gibt es Hinweise in der Literatur zu einem dominanten Sprungmodell?“

F2b: „Gibt es Hinweise in der Literatur zu einem dominanten chaotisch-deterministischen Modell?““

Die Diskussion über nicht-normalverteilte Renditen begann schon mit den Arbeiten von Bachelier (1900). Doch erste wesentliche Beiträge lieferte Mandelbrot (1960), der auf Basis des verallgemeinerten Grenzwertsatzes eine alpha-stabile Verteilung vorschlägt, die die Normalverteilung als Sonderfall umfasst. Die Normalverteilung liegt bei dieser Verteilungsklasse auf einem Alphawert von 2, während 
die empirischen Verteilungen tendenziell bei 1,4 liegen. (Young 2008) Der Vorteil der Verteilung ist die bessere Anpassung der Leptokurtosis und der Fat tails der empirischen Verteilung. Dies führt aber zu dem Nachteil, dass bei Verteilungen mit einem Alphawert kleiner als 2 kein Streuungsmaß zur Berechnung des Risikos existiert. Der eigentliche Streitpunkt ist jedoch die theoretische Interpretation. Denn eine solche Verteilung besitzt eine Langzeitautokorrelation, die eine Zeitreihe auf Basis eines solchen Prozesses prognostizierbar machen könnte. Darin hat auch die eigenwillige Namensgebung als ,deterministisches Chaos“ ihren Ursprung. Diese Prozesse entwickeln sich in vorbestimmten Bahnen, die aufgrund der Sensitivität gegenüber dem Startwert schwierig vorherzusagen sind. Die Theorie ist jedoch umstritten, da für Immobilienmärkte die Markteffizienzhypothese in ihrer starken Form verworfen und die Bedingung der Arbitragefreiheit nicht eingehalten wird.

Untersuchungen zu deterministisch-chaotischen Prozessen wurden für viele Finanzmärkte durchgeführt, auch für Investments in Immobilienmärkten. Die Befunde sind jedoch uneinheitlich und wenig verlässlich, denn die statistischen Tests reagieren äußerst sensitiv auf die Verletzung von Testvoraussetzungen. Zu den aussagekräftigeren Immobilienmarktstudien gehören die von Belaire-French und Opong (2013), Zhou (2011) sowie Assaf (2015), bei denen robustere Tests zum Einsatz kommen. Die Autoren kommen aber nicht zu einer einheitlichen Aussage, ob deterministisches Chaos in den untersuchten Zeitreihen vorhanden ist. Zhou (2011) bestätigt die Hypothese für REIT-Märkte, schließt jedoch nicht aus, dass die Ergebnisse zufällig zustande gekommen sein könnten. Assaf (2015) bestätigt ebenso die Ergebnisse für REIT-Märkte. Belaire-French und Opong (2013) finden lediglich nichtlineare Prozesse, die sich teilweise deterministisch, aber nicht chaotisch verhalten. Selbst wenn zugunsten deterministisch-chaotischer Prozesse in Märkten entschieden wird, stellen Liow und Webb (2008) fest, dass dies keine Rückschlüsse auf direkte Investments in Immobilien zulässt. Daher erscheint die Forderung einer Integration deterministisch-chaotischer Prozesse in die Bewertung von Immobilien und Immobilienportfolios von Wyman et al. (2011) überzogen. Es existieren derzeit keine Befunde, dass diese Ergebnisse auch für direkt gehaltene Immobilienportfolios oder Einzelobjekte zutreffen. Eine unserer Recherchen nach vollständige Auflistung aller Studien zu chaotisch-deterministischen Prozessen im Zusammenhang mit Immobilieninvestitionen findet sich in der nachfolgenden Tab. 3.

Die meisten Tests zum Nachweis deterministisch-chaotischer Prozesse haben eine Gemeinsamkeit: Die Ergebnisse sind sehr sensitiv gegenüber zufälligen Sprüngen in Zeitreihen, was zur zweiten Theorie, der Theorie von Sprungprozessen führt. Ein Sprung verhält sich überproportional gegenüber den Auf- und Abwärtsbewegungen eines normalverteilten Zufallsprozesses. Der Sprung formt dabei die Fat tails, und die Verteilungskurve wird wegen der höheren Häufigkeit extremer Werte gestaucht. Diese Ausreißer (Sprünge) werden häufig mit Klumpenrisiken in Verbindung gebracht und wurden bereits sehr früh von Merton (1976) in einer sogenannten Sprungdiffusion modelliert. Sein Schluss war, dass solche Sprünge bei ausreichender Diversifikation verschwinden, was jedoch nicht zwangsläufig der Fall ist. Es stimmt, dass die Leptokurtosis bei steigender Diversifikation und auch bei einer Ausdehnung des Zeithorizonts abnimmt. Die Exzess-Kurtosis strebt dabei aber viel zu langsam gegen Null. Eine extreme Version dieses Phänomens kann in Tagesren- 
Tab. 3 Untersuchungen zu chaotisch-deterministischen Prozessen einschließlich der Literatur über Testverfahren und Limitationen

\begin{tabular}{|c|c|c|c|c|}
\hline $\begin{array}{l}\text { Studien zu } \\
\text { Chaos }\end{array}$ & $\begin{array}{l}\text { Verwendete } \\
\text { Tests }\end{array}$ & $\begin{array}{l}\text { Literatur zur Testent- } \\
\text { wicklung }\end{array}$ & $\begin{array}{l}\text { Test-Bias } \\
\text { durch/bei }\end{array}$ & Literatur zu Restriktionen \\
\hline $\begin{array}{l}\text { Ambrose et al. } \\
\text { (1992); Newell } \\
\text { et al. (1996); } \\
\text { Liow (2009); } \\
\text { Zhou (2011); } \\
\text { Assaf (2015) }\end{array}$ & $\begin{array}{l}\text { Mod. } \\
\text { R/S-Test }\end{array}$ & Lo (1991) & $\begin{array}{l}\text { Kleine Stich- } \\
\text { probe, Kurz- } \\
\text { zeitautokorre- } \\
\text { lation }\end{array}$ & Teverovsky et al. (1999) \\
\hline Assaf (2015) & V/S-Test & Giraitis et al. (2003) & $\begin{array}{l}\text { Unbekanntheit } \\
\text { des Tests, } \\
\text { Sensitivität } \\
\text { ggü. Sprüngen }\end{array}$ & Assaf (2015) \\
\hline $\begin{array}{l}\text { Newell et al. } \\
(1996)\end{array}$ & GP-Test & $\begin{array}{l}\text { Grassberger und } \\
\text { Procaccia (1983) }\end{array}$ & $\begin{array}{l}\text { Kleine Stich- } \\
\text { probe }\end{array}$ & Ruelle (1990) \\
\hline $\begin{array}{l}\text { Newell et al. } \\
(1996)\end{array}$ & $\begin{array}{l}\text { Lyapunov- } \\
\text { Test }\end{array}$ & Wolf et al. (1985) & $\begin{array}{l}\text { Kleine Stich- } \\
\text { probe, Rau- } \\
\text { schen }\end{array}$ & $\begin{array}{l}\text { Barnett und Serletis } \\
(2000)\end{array}$ \\
\hline $\begin{array}{l}\text { Belaire-French } \\
\text { und Opong } \\
(2013)\end{array}$ & $\begin{array}{l}\text { Mod. } \\
\text { Lyapu- } \\
\text { nov- } \\
\text { Test }\end{array}$ & $\begin{array}{l}\text { Nychka et al. (1992) } \\
\text { Shintani und Linton } \\
(2004)\end{array}$ & $\begin{array}{l}\text { Kleine Stich- } \\
\text { probe }\end{array}$ & - \\
\hline $\begin{array}{l}\text { Wilson und } \\
\text { Okunev (1999); } \\
\text { Elder und Villu- } \\
\text { puram (2012); } \\
\text { Zhou (2011); } \\
\text { Assaf (2015) }\end{array}$ & $\begin{array}{l}\text { Regression/ } \\
\text { Fourier } \\
\text { Regression/ } \\
\text { Wavelet }\end{array}$ & $\begin{array}{l}\text { Geweke und Porter- } \\
\text { Hudak (1983); } \\
\text { Robinson (1995b); } \\
\text { Smith (2005); } \\
\text { Jensen (1999); } \\
\text { Andrews und Gug- } \\
\text { genberger (2003) }\end{array}$ & $\begin{array}{l}\text { Nichtlinearität, } \\
\text { kleine Stich- } \\
\text { probe } \\
\text { Sensitivität } \\
\text { ggü. Sprüngen }\end{array}$ & $\begin{array}{l}\text { Granger und Teräsvirta } \\
\text { (1999); } \\
\text { Diebold und Inoue } \\
\text { (2001); } \\
\text { Granger und Hyung } \\
\text { (2004); } \\
\text { Baillie und Kapetanios } \\
\text { (2007); Rea et al. (2013) }\end{array}$ \\
\hline $\begin{array}{l}\text { Liow (2009); } \\
\text { Elder und Villu- } \\
\text { puram (2012) }\end{array}$ & $\begin{array}{l}\text { LWE/ } \\
\text { Fourier } \\
\text { LWE/ } \\
\text { Wavelet }\end{array}$ & $\begin{array}{l}\text { Fox und Taqqu } \\
\text { (1986); } \\
\text { Robinson (1995a); } \\
\text { Jensen (1999) }\end{array}$ & $\begin{array}{l}\text { Nichtlinearität, } \\
\text { Sprünge, } \\
\text { kleine } \\
\text { Stichprobe; } \\
\text { asymptotisch } \\
\text { abklingend }\end{array}$ & $\begin{array}{l}\text { Granger und Hyung } \\
\text { (2004); } \\
\text { Mikosch und Starica } \\
\text { (2005); } \\
\text { Perron und Qu (2010); } \\
\text { Qu und Perron (2013); } \\
\text { Rea et al. (2013) }\end{array}$ \\
\hline $\begin{array}{l}\text { Zhou (2011); } \\
\text { Assaf (2015) }\end{array}$ & $\begin{array}{l}\text { ELW- } \\
\text { Test }\end{array}$ & $\begin{array}{l}\text { Shimotsu (2010); } \\
\text { Shimotsu (2006) }\end{array}$ & $\begin{array}{l}\text { Kleine Stich- } \\
\text { probe }\end{array}$ & - \\
\hline $\begin{array}{l}\text { Liow (2009); } \\
\text { Zhou (2011) }\end{array}$ & $\begin{array}{l}\text { FIGARCH- } \\
\text { Test }\end{array}$ & Baillie et al. (1996) & $\begin{array}{l}\text { Sensitivität } \\
\text { ggü. Sprüngen }\end{array}$ & $\begin{array}{l}\text { Siehe Regression/ } \\
\text { Fourier; } \\
\text { Mikosch und Starica } \\
\text { (2005) }\end{array}$ \\
\hline $\begin{array}{l}\text { Liow und Webb } \\
(2008)\end{array}$ & $\begin{array}{l}\text { BDS- } \\
\text { Test }\end{array}$ & Broock et al. (1996) & $\begin{array}{l}\text { Sensitivität } \\
\text { ggü. Sprüngen }\end{array}$ & Raydén et al. (1998) \\
\hline $\begin{array}{l}\text { Jirasakuldech } \\
\text { und Emekter } \\
(2012) \\
\text { Newell et al. } \\
(1996)\end{array}$ & - & - & $\begin{array}{l}\text { Vorfilterung } \\
\text { der Zeitreihe }\end{array}$ & $\begin{array}{l}\text { Barnett und Serletis } \\
(2000)\end{array}$ \\
\hline
\end{tabular}

${ }^{\text {a }}$ Die Abkürzungen der Tests stehen für: $R / S$ Range over Scale; V/S Variance over Scale; GP GrassbergerProcaccia; $L W E$ Local Whittle Estimator; ELW Exact Local Whittle; FIGARCH Fractal Integrated Generalized Autoregressive Conditional Heteroscedasticity; BDS Broock-Dechert-Scheinkman 
diten von illiquiden Aktien beobachtet werden, wie beispielsweise die Kursrendite der Areal Immobilien und Beteiligungs- AG verdeutlicht. ${ }^{12}$ Sprünge können in vielen Zeitreihen gefunden werden, nicht nur in Ausnahmesituationen. MaitlandSmith und Brooks (1999) weisen für den NCREIF- und den UK-IPD-Index Sprünge nach. Auch für REITs und Immobilienaktien kann dieses Phänomen eindeutig nachgewiesen werden (Paskelian et al. 2011; Vishwakarma und Paskelian 2012). Allgemein bestehen ausführliche und widerspruchsfreie Ergebnisse zu Sprüngen für verbriefte Immobilieninvestments. Lizieri und Satchell (1997); Lizieri et al. (2012); Kallberg et al. (2002); Krystalogianni und Tsolacos (2004); Liow et al. (2005); Liow und Zhu (2007); Li et al. (2015) sowie Krystalogianni und Tsolacos (2004) weisen ebenso Sprünge in direkten Immobilieninvestments nach. Das Phänomen von Sprüngen kann auch insofern als gesichertes Wissen angesehen werden, da für statistische Tests zur Messung deterministisch-chaotischer Prozesse die Robustheit gegen Sprünge gefordert wird (Baillie und Kapetanios 2007). Aus theoretischer Sicht wird mit Sprüngen die Forderung eines perfekten Hedgings aufgegeben, aber nicht die Forderung der Arbitragefreiheit (Cont und Tankov 2004). Somit bildet dieses Modell im Gegensatz zur Theorie deterministisch-chaotischer Prozesse eine moderatere Erweiterung der neoklassischen Markttheorie.

\section{Die Dynamik innerhalb von Wohnimmobilienportfolios}

Im vorherigen Kapitel wurden die Sprung- und die alpha-stabile Verteilung als Erweiterung der Normalverteilungshypothese diskutiert. Der zweite Schritt der Triangulation widmet sich einer empirischen Auswertung und unterscheidet sich somit stark von der vorherigen Perspektive. Kern der folgenden Analyse ist die Beobachtbarkeit eines Sprung- oder deterministisch-chaotischen Prozesses in den verfügbaren Portfoliodaten. Daher lautet die erste Forschungsfrage:

F3a: „Können die Daten mittels eines Sprungprozesses oder eines alpha-stabilen Prozesses beschrieben werden?"

Alle Sprungprozesse werden durch einen Trend, einen Gauß-Prozess sowie eine Sprungkomponente beschrieben. Dies führt zu zwei Arten von Sprungprozessen: Sprung-Diffusion und reiner Sprungprozess. Sowohl die Normalverteilung als auch die chaotisch-deterministischen Verteilungen sind rein theoretische Positionen. Daher sollte für die Sprungtheorie ebenfalls eine reine Sprungverteilung, wie bspw. die Varianz-Gamma-Verteilung (Cont und Tankov 2004), gewählt werden. In Abb. 3 sind die Quantil-Quantil-Plots für die drei Verteilungen auf Basis der Total Returns (Zeitreihe A) berechnet. ${ }^{13}$ Während die spitze Wölbung von der Varianz-GammaVerteilung und der alpha-stabilen Verteilung gut angenähert werden kann, haben alle drei Modelle Probleme mit der Annäherung der Fat tails. Nach der grafischen

\footnotetext{
12 Siehe hierzu die Analyse des Datensatzes in Kapitel 2.

13 Die Varianz-Gamma-Verteilung wurde mit der BFGS-Methode und der Hessian-Matrix, die alpha-stabile Verteilung wurde mit McCullochs Quantil-Methode für den QQ-Plot angenähert. Als Software kam R Studio 0.99.446 auf Basis von R 3.2.3 und dem Modul VarianzGamma 0.3-1 zum Einsatz.
} 

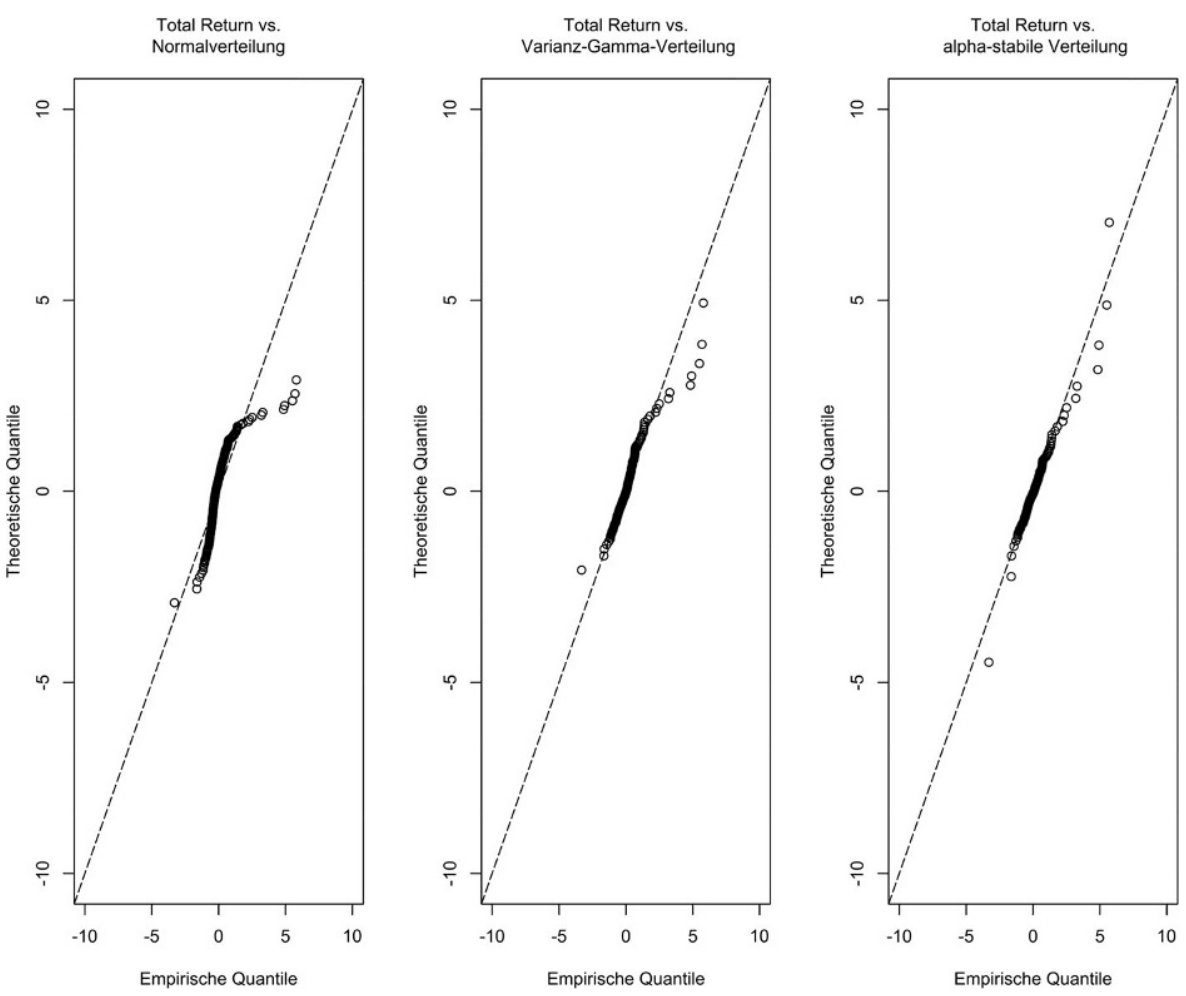

Abb. 3 Quantil-Quantil-Plots zur Anpassung theoretischer Verteilungen an die Total Returns von Zeitreihe A

Analyse fallen die Abweichungen für die Varianz-Gamma-Verteilung am geringsten aus.

Insgesamt können alle drei Modelle statistisch verworfen werden, wobei das Sprungmodell den empirischen Daten noch am ähnlichsten ist. Hier stellt sich die Forschungsfrage:

F3b: „Können Sprünge auch in den aggregierten Zeitreihen beobachtet werden?“

Da der Total Return aus mehreren Variablen besteht, kann auch von einer gemischten Verteilung ausgegangen werden (Armonat 2008, S. 150f.). Es stellt sich dabei die Frage, wodurch die Fat tails einer Verteilung verursacht werden. Eine mögliche Antwort gibt die Analyse der Cash-Inflows des Wohnungsportfolios C1, die in Abb. 4 betrachtet werden. Teil (a) der Abbildung zeigt die Soll-Mieten pro Quadratmeter über einen Zeitraum von 43 Monaten, Teil (b) zeigt das Signifikanzniveau an, das nur für die Monate 15 und 33 unterschritten wird. Die beiden identifizierten Sprünge sind auf einem Niveau von $p$ kleiner als 5\% signifikant. Das Signifikanzniveau wurde mittels eines rollierenden Mittelwertvergleichs ${ }^{14}$ auf Basis der SollMieten pro Quadratmeter und Objektebene einer Periode (Querschnittsvergleich)

14 Der überwiegende Teil der monatlichen Perioden zeigt eine deutliche Abweichung der Soll-Mieten von der Normalverteilung an. Daher wurde für den Mittelwertvergleich der Wilcoxon-Rangsummen-Test 

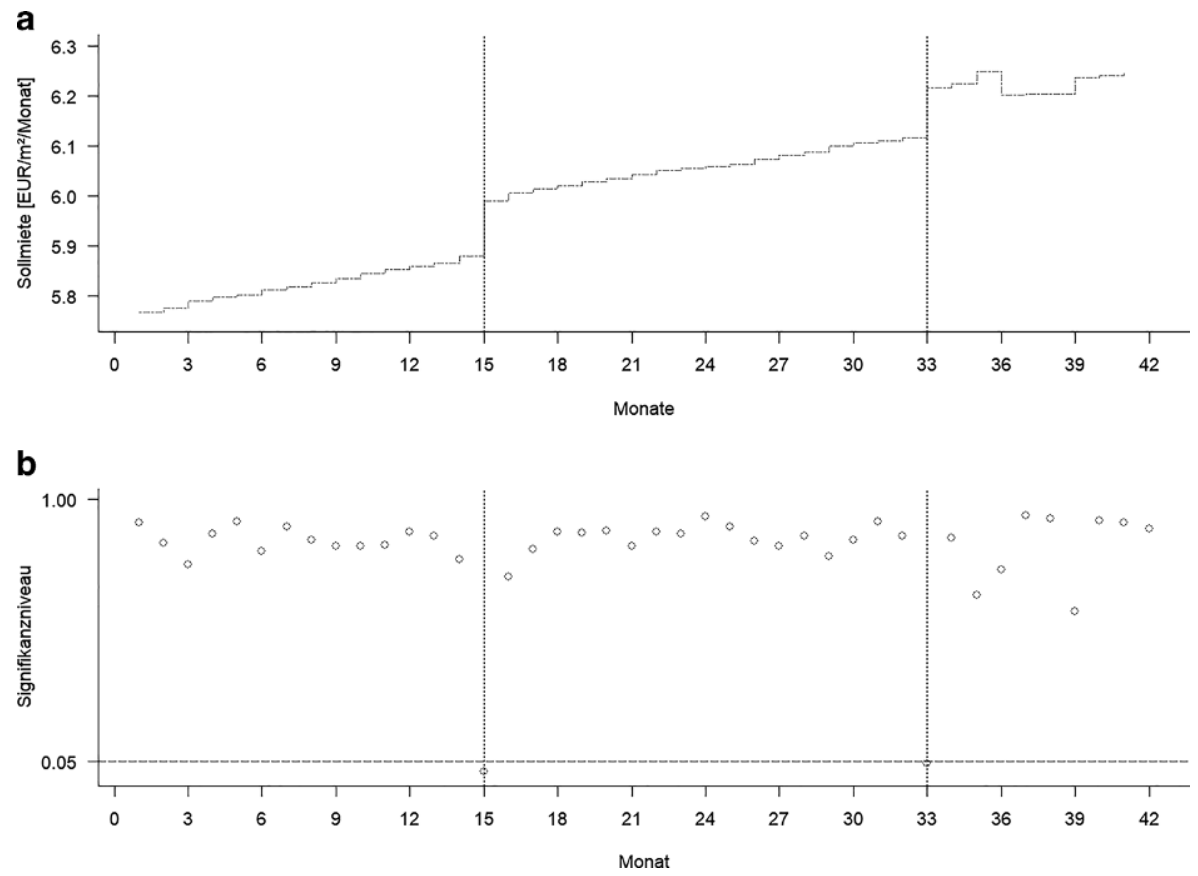

Abb. 4 Soll-Mieten und Signifikanzniveau des Portfolios C3

errechnet. Die Steigung der Zeitreihe entspricht dem Augenschein nach einem deterministischen Trend mit stufenförmiger (sprunghafter) Entwicklung. Die beiden signifikanten Sprünge sind durch Mieterhöhungen entstanden, wie die Befragung des Datenlieferanten ergab. Die Abwärtsbewegung am Ende der Zeitreihe ist laut dem Datenlieferanten auf eine Mietsenkung durch politischen Druck zurückzuführen.

Solche Muster können durch vielfältige Gründe hervorgerufen werden, die im Folgenden beispielhaft illustriert werden. Folgen wir der am Anfang gewählten Systematik, wäre ein exogener Grund durch regional unterschiedlich steigende Marktmieten gegeben. Variierende Abstände (Frequenzen) zwischen den Mieterhöhungen können einen Sprung auslösen, wenn der Bestand entsprechende Konzentrationen aufweist. Hierdurch würden Mieterhöhungen gehäuft in einer Periode auftreten und einen Sprung in der Zeitreihe sichtbar machen. Dies dürfte zwar selten vorkommen, das Beispiel der Hansaimmobilia zeigt aber, dass dieses Szenario beobachtet werden kann. Ein endogener Grund sind Arbeitsabläufe, die sich auf die Zahlungsflüsse auswirken, wie die Sammelmieterhöhungen und -senkungen des Portfolios C3 zeigen. Allerdings sind sie in diesem Umfang ungewöhnlich. Es ist nicht unüblich Mietanpassungen über mehrere Wirtschaftseinheiten zusammenzufassen, aber eine Erhöhung für ca. 50\% des Bestandes sticht in der Analyse heraus.

gewählt, da dieser Test gegenüber Abweichungen von der Normalverteilung robust ist und dadurch Fehler zweiter Art reduziert werden. 


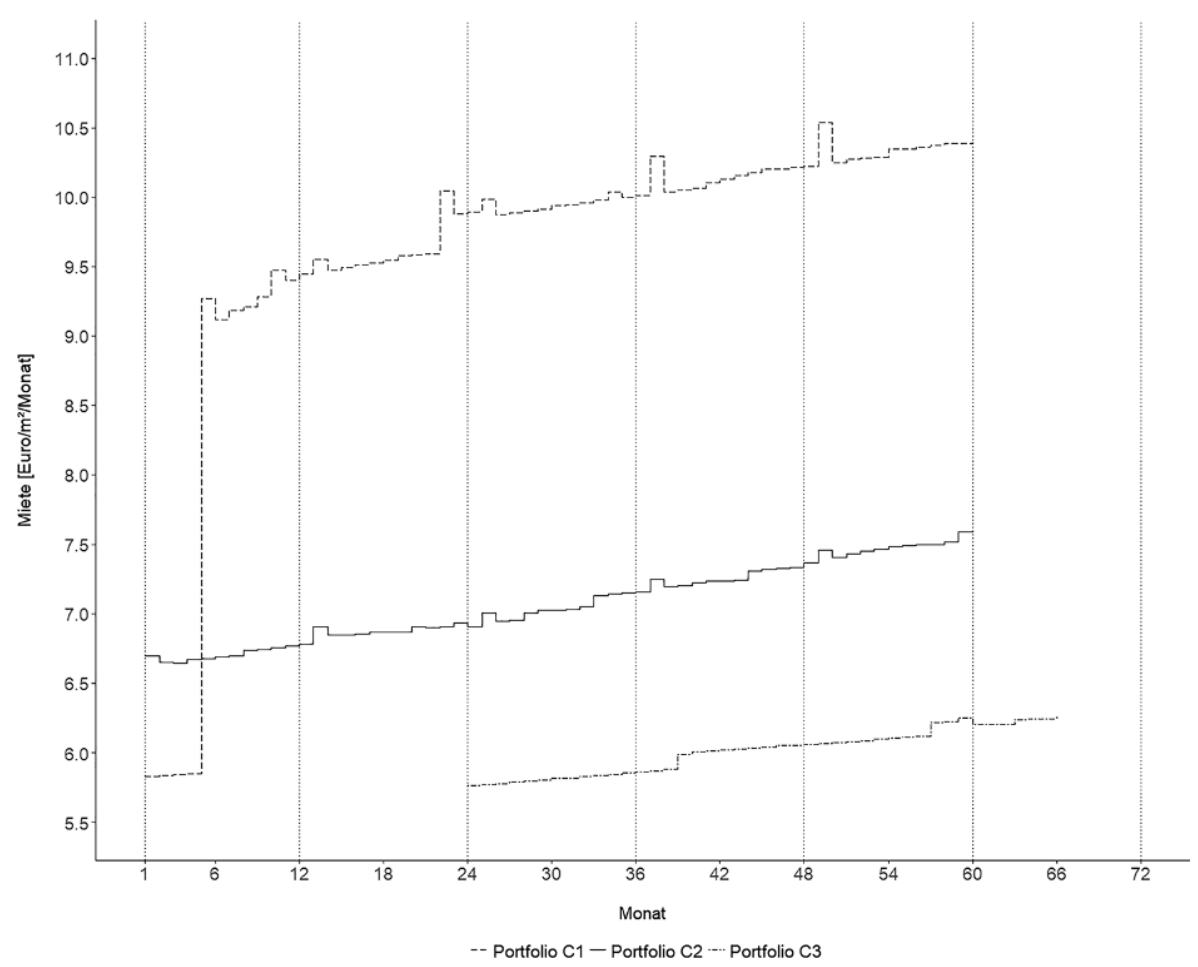

Abb. 5 Soll-Mieten von drei Portfolios im Zeitverlauf

Auch in den Daten der Wohnungsportfolios C1 und C2 können Sprünge optisch identifiziert werden (siehe Abb. 5). ${ }^{15}$ Der große Sprung im Portfolio C1 ist nach Aussage des Datenlieferanten auf eine Mieterhöhung nach einer Teilmodernisierung des Portfolios zurückzuführen. Die turmartigen Gebilde am Anfang eines jeden Jahres - also in den Monaten 13, 25, 37 und 49 -, rühren aus wenigen gewerblichen Mietverträgen her, die jährlich oder quartalsweise im Voraus bezahlt werden. Dies können beispielsweise Verträge zu Mobilfunkmasten, Werbetafeln oder sonstige Gewerbemietverträge mit jährlichem Zahlungsturnus sein. Die Miete steigt bei Einzahlung in dem jeweiligen Monat und sinkt im nächsten Monat wieder auf das Ausgangsniveau. Die Gründe für solch ein sprunghaftes Verhalten können somit vielfältiger Natur sein, sind aber auf bestimmte Maßnahmen der Geschäftstätigkeit und „Handelsbräuche“ zurückzuführen.

Die Sprünge nehmen für kleine Portfolios grundsätzlich zu, was durch die geringere Diversifikation begründet werden kann. Das Portfolio C3 ist wesentlich größer als die Portfolios C1 und C2. Es ist ersichtlich, dass die Anzahl der Mietverträge glättend auf die Zeitreihe einwirkt, wenn eine hohe Anzahl an Wohneinheiten vor-

15 Für die Portfolios C1 und C2 kann die Analyse der Sprünge nur optisch erfolgen, da kein geeigneter Test für die geringe Datenmenge existiert. Dadurch wird die Belastbarkeit der Auswertung natürlich eingeschränkt, aber die Sprünge treten im Gegensatz zum Portfolio C3 optisch deutlich hervor. 
liegt, wie es auch in den Zeitreihen des GdW (2016) zu sehen ist. Dieser Effekt ist auch als Granularität bei Fremdkapitalinstrumenten oder naive Diversifikation bei Eigenkapitalinstrumenten bekannt (Benartzi und Thaler 2001; Norden et al. 2016). Bei Gewerbeimmobilien scheint auch die Höhe der Mietzahlungen im Verhältnis zu den übrigen Mietzahlungen relevant zu sein. Wenn der Anteil der Soll-Miete geringer ausfällt, kann dieser Effekt durch das allgemeine Mietniveau des Portfolios kompensiert werden, da der prozentuale Anteil der gewerblichen Mieten abnimmt (vgl. bspw. Portfolio C1 und C2). Auch scheint die Soll-Mieten-Entwicklung kein reiner Zufallsprozess zu sein, da zu viele Regelmäßigkeiten in den Zeitreihen sichtbar sind. Abschließend muss festgehalten werden, dass die Zeitreihen optisch weder einem Sprungprozess noch einem chaotisch-deterministischen Prozess ähneln.

In Abb. 6 sind die kumulierten Kosten für (a) die geplante Instandhaltung, (b) die Modernisierung von Immobilien und (c) die ungeplante Instandhaltung der Portfolios C1 und C2 abgebildet. Die geplante Instandhaltung und die Modernisierung weisen ebenfalls sprunghafte Muster zum Jahresende hin auf. Vermutlich ist die Entwicklung auf saisonale Muster und auf Budgetierungs- und ähnliche Besonderheiten in den betrieblichen Prozessen zurückzuführen. Die abnehmende Steigung des Trends für die geplante Instandhaltung und Modernisierung ist wahrscheinlich bedingt durch den aufholenden Effekt der durchgeführten Bauleistungen.

Im Kontrast zu den aktiv-gemanagten Zeitreihen (geplante Instandhaltung, Modernisierung) können bei der ungeplanten Instandhaltung optisch keine deutlichen Sprünge identifiziert werden. Die Leptokurtosis deutet aber darauf hin, dass wohl Sprünge enthalten sind. Insgesamt weist die ungeplante Instandhaltung jedoch einen relativ konstanten Trend auf, der nur kleine, vermutlich zufallsbedingte Variationen aufweist. Dies ist wenig überraschend, da der Name ,ungeplante Instandhaltung“ bereits auf den Zufallscharakter hindeutet. Wie im weiteren Verlauf des Artikels noch gezeigt wird, sollte man aus dem Kurvenverlauf nicht folgern, dass Sprünge lediglich durch planerische Aktivitäten ausgelöst werden. Weiterhin müsste sich aufgrund der Modernisierungs- und Instandhaltungsmaßnahmen auch ein aufholender Effekt in der ungeplanten Instandhaltung zeigen, der hier jedoch fehlt. Eine plausible Erklärung ist schwierig, aber es könnte mit dem Prozess der Budgetierung zusammenhängen, der die Zeitreihe von den restlichen Maßnahmen ,entkoppelt“. Dem Augenschein nach erscheint es sinnvoller, Prognosen für die ungeplante Instandhaltung mittels der kumulierten Zeitreihe vorzunehmen, anstatt absolute monatliche Auszahlungen zu verwenden.

Die Ergebnisse zeigen, dass betriebliche Entscheidungen die Sprünge in den Zeitreihen und damit die Abweichungen von der Normalverteilung zumindest teilweise plausibel erklären können. Diese Sprünge treten zum Teil in periodischen Abständen auf und können kaum als zufällig betrachtet werden. Hieraus folgt, dass ohne Korrekturen für planmäßige, periodische und saisonale Muster eine Verteilung nicht sinnvoll für die Risikomessung verwendet werden kann. Bei manchen Zahlungsströmen besteht weniger Unsicherheit, beispielweise bei Mieterhöhungen, da die Maßnahme im Voraus absehbar ist und die Miete danach normalerweise mit derselben Regelmäßigkeit eingeht wie vorher. Auf der anderen Seite ändern sich die Mieteinnahmen nicht, sobald eine Ratingagentur die Bonität eines Mieters herabstuft und die Unsicherheit der zukünftigen Cashflows demnach zunimmt. Diese 

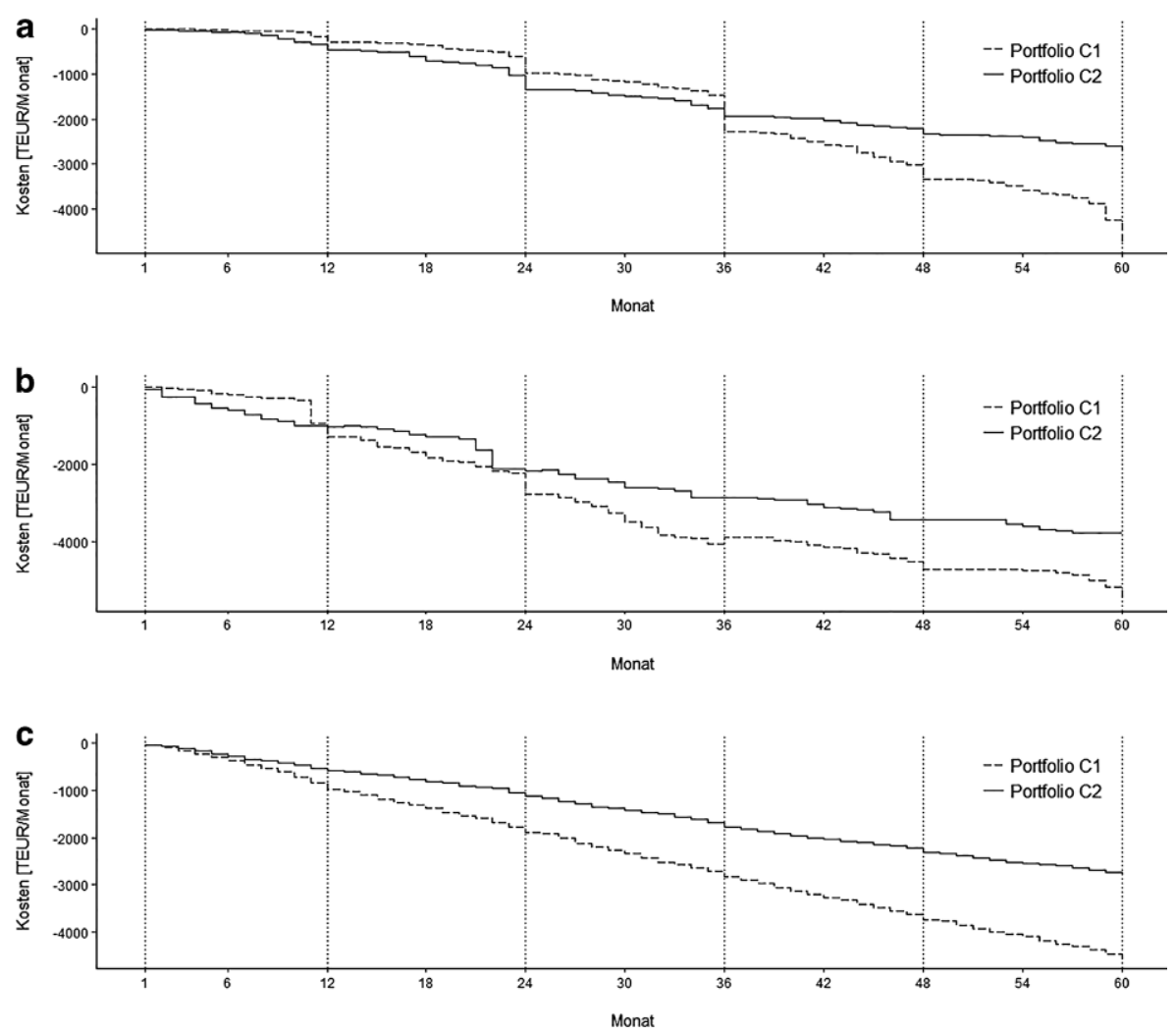

Abb. 6 Kumulierte Kostenverläufe für drei Kostenarten für zwei Portfolios

beiden Beispiele weisen auf ein grundsätzliches Problem hin: Es gibt für den Begriff „Risiko“ keine einheitliche Definition, was die Konstruktion geeigneter, objektiver Risikomaße schwierig macht.

Auch der Erwartungswert scheint nicht geeignet zu sein, da ein Mittelwert die Planung des aktiven Managements nicht abbildet. Jedoch ist diese Planung als implizite Information im Kaufpreis der Immobilie enthalten. Weiterhin ist es nachvollziehbar, dass Mieterhöhungen zu Sprüngen in den Einnahmen führen. Eine andere Frage ist, ob ein Sprung ein Risiko - also eine Abweichung vom Mittelwert - darstellt. Hier kann man sich auf den Standpunkt stellen, dass nicht die Erhöhung der SollMiete das Risiko darstellt, sondern die Unsicherheit, ob die Ist-Miete der Soll-Miete entsprechen wird. In manchen Fällen können Mieterhöhungen nur mit Verzögerung oder gar nicht durchgesetzt werden. Daraus folgt ein höheres Liquiditätsrisiko, wofür der Sprung der Soll-Miete jedoch ein unpräziser Stellvertreter ist. Als Ausgangsbasis für ein Risikomaß auf Portfolio- bzw. Objektebene scheint die Differenz aus Planung und Realisation angebrachter. Abschließend können die Ergebnisse noch einmal der folgenden Tab. 4 entnommen werden. 
Tab. 4 Zusammenfassung der Ergebnisse aus den Abb. 2, 3, 4 und 5

\begin{tabular}{ll}
\hline Abb. & Ergebnisse \\
\hline 2 & Die VG-Verteilung und die alpha-stabile Verteilung können die Exzess-Kurtosis der empiri- \\
& schen Verteilung besser anpassen als die Normalverteilung. \\
& Die VG-Verteilung kann die Fat tails der empirischen Verteilung ebenfalls besser als eine \\
& Normalverteilung anpassen, während die alpha-stabile Verteilung die Fat tails um bis zu \\
& 200\% überschätzt. \\
& In den Soll-Mieten konnten Sprünge in den Monaten 15 und 33 nachgewiesen werden, die \\
& signifikant auf einem Niveau von $p \leq 5 \%$ sind. Dies entspricht zwei Sprüngen innerhalb \\
& eines Zeitraums von 18 Monaten, die durch Sammelmieterhöhungen entstanden. \\
& Für die Portfolios C1 und C2 konnten in den Monaten 13, 25, 37 und 49 optisch Sprünge \\
& in den Soll-Mieten festgestellt werden, die jedoch kein erkennbares Muster aufweisen. Des \\
& Weiteren existieren für das Portfolio C3 signifikante Sprünge (s. oben) in den Soll-Mieten. \\
& Weiterhin sind periodische Zahlungsspitzen zum Jahresanfang und -ende in den Portfolios \\
& C1 und C2 sichtbar, die bspw. auch Sprünge (C1; s. Monat 5 und 22) überlagern. \\
& Die Kostenpositionen Modernisierung und geplante Instandhaltung weisen optisch ebenfalls \\
& periodische Sprünge auf, die aber nicht ausschließlich auf das Jahresende fallen. \\
& Für die ungeplante Instandhaltung (c) können optisch keine Sprünge festgestellt werden. \\
& Die Sprünge aus den Eingangsvariablen des Total Returns scheinen sich in den Total Return \\
& zu übertragen. \\
& Diese (deterministischen) Sprünge scheinen der Grund zu sein, dass die VG-Verteilung \\
besser an die empirischen Daten angepasst werden kann.
\end{tabular}

\section{Die Bewertung von Risiken bei Nicht-Normalverteilung}

Eine sehr häufige Perspektive, auch in der immobilienwirtschaftlichen Literatur, ist die Betrachtung des Risikos als eine eindeutige, objektive und quantifizierbare Größe. Dieser Ansatz geht auf die Erwartungsnutzentheorie und die moderne Portfoliotheorie zurück (Markowitz 1952; von Neumann und Morgenstern 1944). Allerdings ist es wenig überraschend, dass dieses Entscheidungsverhalten in empirischen Untersuchungen nicht festgestellt werden kann (Kahneman 2016; Bals und Wellner 2016). Dies liegt auch daran, dass Risiko als soziales Konstrukt betrachtet werden muss (Luhmann 1997; Slovic 1987), wodurch es eine mehrdimensionale, subjektive und schlecht quantifizierbare Größe wird. Die Vertreter dieser Perspektive führen neue Variablen ins Feld, beispielsweise die wahrgenommene Eintrittswahrscheinlichkeit oder die antizipierten Emotionen im Schadensfall. Versucht man beide Ansätze in Einklang zu bringen, muss man akzeptieren, dass man mit objektiven und subjektiven Risikomaßen nur jeweils einen Teil des Risikos bewerten kann und noch keine überzeugende Methode der gemeinsamen Bewertung gefunden wurde. Im vorherigen Kapitel wurden Gründe für Sprünge identifiziert, die beispielsweise durch Sammelmieterhöhungen oder kostenintensive Einzelmaßnahmen entstehen können. Eine vollständige Inventarisierung solcher Gründe ist durch die Fülle an Möglichkeiten ausgeschlossen, auch wenn eine intensivere Forschung diesbezüglich wünschenswert ist. Daher wird in der letzten Perspektive der Triangulation mittels 
unterschiedlicher Quellen, Logik und Interviews eine Taxonomie ${ }^{16}$ als Begründung gewagt, die die komplexe empirische Verteilung zu formen scheint. Die dazugehörige Forschungsfrage lautet:

F4: „Existieren Charakteristika anhand derer die beobachteten Muster kategorisiert werden können?“

Ein Kernproblem bei der Risikobewertung bildet die subjektive Wahrnehmung der Beteiligten, die die Risikokommunikation erschwert. Callahan (2002) sowie Smithson und Simkins (2005) illustrieren dies anhand von Hedging-Strategien, die vom Aktienmarkt durch Preisabschläge bestraft werden. Ähnliche Beispiele können auch in der Immobilienwirtschaft gefunden werden. Eine klassische Gegenstrategie liefern die Interviewpartner 1 und 2 aus dem Asset- und Risikomanagement: Eine allzu klare Kommunikation sei demnach nicht immer gewünscht, da sie den Verfasser angreifbar machen könnte. Ein weiteres Beispiel findet sich in der Immobilienbewertung: Gutachten werden in der Regel so verfasst, dass der Immobilienwert oberhalb des ,wahren“ Wertes einer Immobilie liegt (Crosby und Hughes 2011; Hansz und Diaz III 2001; Chinloy et al. 1997). Interviewpartner 3 (2016) ergänzt diese Ergebnisse aus seiner Sicht mit der ökonomischen Begründung, dass etwaige Diskussionen zu höherem Aufwand oder zur Unzufriedenheit des Kunden führen können. Dies ist ein grundsätzliches Principal-Agent Problem, das selbst durch eine geeignete Managementstruktur, die Interessengleichheit anstrebt, nicht restlos aufgelöst werden kann. An dieser Stelle kann zwar keine überzeugende Methode zur Lösung all dieser Probleme vorgestellt werden, aber es werden Überlegungen präsentiert, wie Risiken unter Beachtung der Nicht-Normalverteilung der Renditen bewertet werden können. Menschen sind durchaus in der Lage, anhand von Kennzahlen subjektive Prognosen zu erstellen, die vergleichbar mit der Präzision statistischer Ergebnisse sind (Lawrence et al. 2006). Die Grundlage könnten die folgenden Kennzahlen zur Bewertung von Risiken bilden, die anscheinend mit der komplexen Form empirischer Verteilungen in Verbindung stehen.

Lage: Die Bewertung durch einen Gutachter stellt eine stichtagsbezogene Einschätzung der zukünftigen Entwicklung einer Immobilie dar. Erhöht sich der Wert einer Immobilie, liegt dieser Betrag nicht liquide auf einem Konto vor, sondern der Mehrwert wird erst mit dem Verkauf realisiert. Daher entspricht der Total Return nicht der tatsächlich erwirtschafteten Rendite, sondern einer geschätzten Rendite. Im vorherigen Absatz wurde bereits darauf hingewiesen, dass Fluktuationen im Total Return enthalten sein können, die kein Risiko darstellen und damit die Messung verfälschen. Geeigneter als eine auf einer Bewertung basierende Kennzahl scheint der Vergleich mit den tatsächlich erwirtschafteten Größen zu sein. Die Differenz zwischen Soll- und Ist-Rendite ergibt eine Wahrscheinlichkeitsverteilung, die die Abweichung von tatsächlichen Erwartungen wiedergibt. Wenn sich Soll- und IstWert decken, existiert kein Risiko. Entstehen Abweichungen, erlaubt dies eine Auf-

\footnotetext{
16 Diese Vorgehensweise ist weniger an einen strikten Beweis orientiert als an dem Versuch, wiederkehrende Muster zu identifizieren und zu kategorisieren. Die Bedeutung der Interviews soll dabei nicht dem Beweis dienen, sondern als Hintergrundinformationen eine bessere Einordnung ermöglichen.
} 
spaltung in antizipierte und nicht-antizipierte Risiken, ähnlich der Methodik zur Prognose der Inflation (Dornbusch et al. 2003, S. 141). Sehr wahrscheinlich wird diese Kennzahl ebenfalls von einer Normalverteilung abweichen. Sie bietet aber den Vorteil, dass sie ohne die Annahme des homo oeconomicus auskommt. Aus diesem Grund wird das Lagemaß als alleinige Kennzahl auch nicht ausreichen, so dass es weiterhin mehrerer Kennzahlen bedarf. Die folgenden Konzepte scheinen hierfür allenfalls ein geeigneter Startpunkt zu sein.

Granularität: Die einfachste Form der Diversifikation ist eine gleichmäßige Verteilung des Vermögens auf möglichst viele unabhängige Vermögensgegenstände. Diese Strategie umfasst die elementaren Konzepte „Anzahl der Assets“ sowie „gleiche Gewichtung der Assets" und ist unter dem Namen naive Diversifikation oder „1/N-Regel“ bekannt. Obwohl die naive Diversifikation die Korrelationen zwischen den Vermögensteilen vernachlässigt, scheint sie sich positiv auf die Schwankungen des Portfolios auszuwirken (DeMiguel et al. 2009; Wellner 2003). Dieser Effekt kann auch in den Cashflows der zuvor untersuchten Wohnungsportfolios festgestellt werden. Vermutlich liegt es daran, dass selten kausale Zusammenhänge zwischen Wohnungsmietverträgen bestehen. In Gewerbeimmobilienportfolios, in denen Mietverträge mit festen Laufzeiten dominieren und die Miethöhen sehr unterschiedlich sein können, sieht das anders aus. Das Beispiel der HansaInvest (2011, S. 9) zeigt, dass die unterschiedlichen fixierten Laufzeiten dazu neigen „Klumpen“ zu bilden. Interviewpartner 2 (2016) ist der Meinung, dass es praktisch unvermeidbar ist, dass sich Mietvertragsausläufe in bestimmten Jahren kumulieren. Dieser zweite Teil der Strategie verdient eine gesonderte Betrachtung und kann unter dem Begriff „Homogenität" zusammengefasst werden.

Homogenität: Als Grund für nicht-normalverteile Renditen spielen Ausreißer und Klumpen eine elementare Rolle. Diese können sich durch einmalige große Ereignisse oder eine Vielzahl kleiner Ereignisse zum selben Zeitpunkt bilden. Je höher die Homogenität von Zahlungsgrößen, desto mehr nähern sich die Zahlungsgrößen der 1/N-Regel an, der Gewichtung zu gleichen Teilen. Dabei muss festgehalten werden, dass so viele Klumpenrisiken entstehen können, wie es Merkmale in einem Portfolio gibt. Zur Messung der Homogenität existieren mehrere absolute Konzentrationsmaße, wie der Rosenbluth-Index, sowie relative Konzentrationsmaße bzw. Ungleichverteilungsmaße, wie der Gini-Koeffizient (Eliazar und Sokolov 2010; Hirschman 1964; Rosenbluth 1955). Bei Kreditverträgen - die Ähnlichkeiten zu Mietverträgen aufweisen - wird häufig der Herfindahl-Hirschman-Index (Agarwal et al. 2011) verwendet, der eine konservative Kompensation für die Granularität eines Portfolios darstellt. Er entspricht dem Korrekturfaktor vom arithmetischen Mittelwert zum gewichteten Mittelwert. Diese Maßzahlen haben allerdings den Nachteil, dass sie zeitpunktbezogen sind und dynamische Effekte nicht berücksichtigen (Huschens und Stahl 2004). Gerade diese zeitliche Komponente kann die Bildung von Klumpenrisiken maßgeblich beeinflussen, was unter dem Begriff Frequenz zusammengefasst werden kann. 
Frequenz: Sowohl Granularität als auch Homogenität eines Portfolios sind auf einzelne Zeitpunkte oder einen normierten Zeitraum beschränkt. Selbst unter Ausschluss jeglicher Einwirkungen ist die Homogenität nicht zwangsläufig konstant. Dies liegt an der Frequenz von Ereignissen im Zeitverlauf, wie das Beispiel des Hansaimmobilia-Fonds mit mehr als $30 \%$ Mietvertragsausläufen zeigt (Hansainvest 2011, S. 9). Ein solches Problem bildet die im gewerblichen Immobilienbereich übliche Kennzahl „Weighted Average Lease Term (WALT)“ nicht ab. Der WALT gibt zwar Auskunft über die Unsicherheit der zukünftigen Cashflows, aber nicht über dessen Schwankungen. Dies zeigt ein kurzes Beispiel: Ein 10-Jahres- und ein 5-Jahres-Mietvertrag gleicher Größe ergeben einen WALT von 7,5 Jahren. Zwei gleich große Mietverträge über 7,5 Jahre ergeben auch einen WALT von 7,5 Jahren. Das Leerstandsrisiko beträgt im ersten Fall 50\%, weil die Mietverträge zu unterschiedlichen Zeitpunkten auslaufen, im zweiten aber $100 \%$, da beide Mietverträge gleichzeitig auslaufen. Eine Möglichkeit zur Lösung dieses Problems wäre die Berechnung der Streuung des WALT, die bei größerer Streuung stabilere Cashflows verspricht, wie das obige Beispiel verdeutlicht. Gleiche Beispiele können auch für Instandhaltungs- und Instandsetzungskosten konstruiert werden, wie ähnliche Überlegungen von Swiss Finance \& Property AG $\left(2015\right.$, S. 8) zeigen. ${ }^{17}$

Dies zeigt noch eine weitere interessante Eigenschaft, die in der Literatur als glättender Effekt bezeichnet wird (Hamilton et al. 2006; Hendershott 2002; Myer und Webb 1994, 1993). Eine Ausdifferenzierung von Laufzeiten in Verbindung mit der Erhöhung der Granularität wirkt dämpfend auf das Risiko durch Marktänderungen, wofür sich in der immobilienwirtschaftlichen Praxis mehrere Belege finden. Im Bereich der Kreditfinanzierung gibt es beispielsweise die Strategie, Immobilien mit mehreren Krediten unterschiedlicher Laufzeit zu finanzieren, um Zinsschwankungen zu dämpfen (Ledergerber 2012; Norden et al. 2016). Für das Segment des studentischen Wohnens stellt Rottwilm (2011, p. 2) fest, dass die kurzen Mietverträge zu einer besseren Anpassung an den Markt beitragen. Hamilton et al. (2006) stuft die in Großbritannien üblichen langen Mietvertragslaufzeiten in Verbindung mit der Upward-Only-Klausel als eine Art Absicherung gegen Marktfluktuationen ein. In Bezug auf den Cashflow scheint dieser Mechanismus aber nicht bewusst für das Risikomanagement eingesetzt zu werden. Interviewpartner 1 (2016) und Interviewpartner 2 (2016) stufen diese Maßnahme unabhängig voneinander als eher ungeeignet ein, da eine Anpassung über Neuvermietungen aus Kostengründen vermieden werden sollte, wenn die Anpassung auch durch eine Prolongation des Vertrags vereinbart werden kann $^{18}$.

Die präsentierten Konzepte bieten sowohl Vor- als auch Nachteile. Die Ersetzung des Erwartungswertes durch ein Planungskonstrukt kann ein Vorteil sein, da die Planung durch das Feedback Learning und das Debiasing verbessert werden kann (Karelaia und Hogarth 2008; Lawrence et al. 2006; Croskerry et al. 2013).

\footnotetext{
17 Es existieren auch ähnliche Überlegungen im Bereich der Konjunkturtheorie und des Hochfrequenzhandels. Aufgrund der teils extremen Komplexität dieser Methoden und eventueller Zirkelschlüsse wurde diesen Parallelen im vorliegenden Aufsatz nicht nachgegangen.

18 Diese Sicht ist natürlich subjektiv, auch wenn sich die Meinungen unabhängig voneinander decken. Eine Untersuchung, die einen Vergleich dieser Grenzkosten anstellt, ist den Autoren leider nicht bekannt.
} 
Des Weiteren können die Konzepte der Granularität, Homogenität und Frequenz leichter in Maßnahmenkataloge transponiert werden als Risikomaße wie der Valueat-Risk. Ein weiterer Vorteil ist auch die Übertragbarkeit auf sehr unterschiedliche Bereiche wie Kosten oder Bilanzpositionen. Zudem sind die Kennzahlen mittels Monte-Carlo-Simulation dynamisch abschätzbar, so dass dies ein echter ,forwardlooking approach“" wäre (Wheaton et al. 2001), was allerdings mit erheblichem Rechenaufwand verbunden wäre sowie zusätzliche Kenntnisse in der Ermittlung und Interpretation komplexer Kennzahlen erfordern würde. Ein weiterer Nachteil ist die Aufspaltung des Risikos in mehrere Kennzahlen. Es ist nicht absehbar, ob sich diese Kennzahlen in ein einheitliches Kennzahlensystem zusammenführen lassen. Es ist somit unklar, wie sich die vorgeschlagenen Maße zu den in der Praxis verfolgten Investitions- und Risikomanagementstrategien verhalten. Jedoch bieten die vorgestellten Konzepte eine alternative Herangehensweise zu den üblichen kapitalmarktheoretischen Ansätzen, deren Grenzen mittlerweile hinlänglich bekannt sind. Es sind jedoch weitere Untersuchungen notwendig, um die gewonnenen Einsichten in ein theoretisches Konstrukt einzubetten und auf Widerspruchsfreiheit und Vollständigkeit zu testen.

\section{Zusammenfassung und Ausblick}

Ziel des Artikels war es, ausgewählte Gründe für Abweichungen von der Normalverteilung bei deutschen Wohnimmobilien zu untersuchen und Hinweise auf neue Risikomanagementansätze zu liefern. Dafür wurden drei unterschiedliche Perspektiven gewählt, bei denen teilweise unterschiedliche Daten und Tests zum Einsatz kamen. Die Untersuchung baut auf einer Literaturrecherche, der Auswertung von Mietvertrags-, Rendite- und Börsendaten sowie Experteninterviews auf. Insbesondere die umfangreichen Datensätze zu den drei Wohnungsportfolios gewähren detaillierte Einblicke in die Dynamik von Immobilienbeständen. Durch die eingeschränkte Validität der Untersuchung müssen die Ergebnisse allerdings sehr vorsichtig interpretiert werden, was ein typischer Nebeneffekt explorativer Forschung ist. Es ist den Autoren ebenfalls bewusst, dass eine Reproduktion der Ergebnisse durch die Vertraulichkeit der Daten ausgeschlossen ist. Die Falsifizierung dieser Untersuchung ist durch andere Datensätze mit Renditen, Cashflows und Kosten aber nicht vollständig ausgeschlossen. Trotz dieser Limitationen sind einige wichtige Ergebnisse erzielt worden, die hier nochmals zusammengefasst werden.

Die Abweichungen von der Normalverteilung scheinen eher durch sprungartige Muster getrieben zu sein. Die Gründe für die Sprünge können nicht abschließend geklärt werden, da unendlich viele unterschiedliche Ereignisse vorstellbar sind. Jedoch können diese Gründe klassifiziert werden. Erstens - Granularität: Die Normalverteilung ist eine stetige Wahrscheinlichkeitsverteilung, wobei Immobilienportfolios durch die Anzahl ihrer Immobilien, Mietverträge und weiterer Merkmale eher einer diskreten Entwicklung folgen. Je kleiner ein Portfolio ist, desto stärker wächst die Intensität des Sprungs, und die Diskretheit des Merkmals tritt immer deutlicher hervor. Dies kann bei der illiquiden Aktie, bei Sammelmieterhöhungen in Portfolio C3 oder der Modernisierung in Portfolio C1 beobachtet werden. Zweitens - Ho- 
mogenität: Sprünge können auch auftreten, wenn zwar viele Elemente vorhanden sind, diese aber nicht gleichverteilt auftreten. Hier können die gewerblichen Mietverträge in den Portfolios C2 und C3 angeführt werden, die durch ihr Volumen und ihre besondere Sollstellung Sprünge im Portfolio verursachen. Drittens - Frequenz: Die Frequenz von Ereignissen ist eine weitere moderierende Größe. Die Frequenz kann auf unterschiedliche Weise zu Sprüngen führen. (a) Inaktivität innerhalb eines Zeitverlaufs erhöht die Leptokurtosis, da die sich nicht ändernden Werte redundant sind. Die anschaulichsten Beispiele sind die illiquide Aktie und der Mieten-Cashflow einer einzelnen Wohnung. Letzteres ist insofern interessant, da das Mietrecht die Frequenz besonders stark bei Wohnungsmieten reglementiert. (b) Weiterhin könnten Frequenzüberschneidungen zu Sprüngen führen, da unterschiedlich fixierte Mietvertragslaufzeiten zu sog. ,Klumpen“ führen können, die aber von einer Diversifikation auf Objektebene unabhängig sind. Organisatorische Gründe scheinen ebenfalls eine Rolle zu spielen, wie das Beispiel der Sammelmieterhöhung zeigt. Auch Skaleneffekte sind vorstellbar, die zu einer gesammelten Abarbeitung von Maßnahmen beitragen können. Die Untersuchung zeigt auch, dass die Sprünge nicht rein zufällig zu sein scheinen, da periodische Muster in den Daten auftreten. Daher müssen Sprünge auch nicht per se ein Risiko darstellen, insbesondere wenn Planungsaspekte diese Sprünge formen.

Die Risikomessung sollte um diesen Planungsteil bereinigt werden, da ein Immobilien-Assetmanager im Gegensatz zu einem Wertpapier-Assetmanager das Investment aktiv steuern kann. Des Weiteren verfälschen Wertgutachten die Risikomessung durch ein Modellrisiko, sobald der wertermittlungsbasierte Total Return verwendet wird. Diese Kennzahl schadet bei der Risikomessung mehr als sie nützt, auch wenn der Total Return durchaus seine Berechtigung bei der Performancemessung hat. Für die Risikomessung ergibt sich daraus die Notwendigkeit einer Neuberechnung von Lagemaßen, die auf Basis von Plan-Ist-Differenzen gebildet werden sollten. Dies würde eine klar abgegrenzte verhaltensorientierte (subjektive) Komponente integrieren, die einer objektiven Größe gegenübergestellt wird. Insgesamt bedarf es neuer Kennzahlen und einer sorgfältigen Auswahl „harter“ Daten. Die drei aufgeführten Kategorien Granularität, Homogenität und Frequenz sollten helfen, neue Denkansätze zu entwickeln. Entsprechende Kennzahlen liegen für diese Kategorien bereits teilweise vor.

Insgesamt scheint es geboten, den Risikobegriff für die Immobilienwirtschaft auf den Prüfstand zu stellen. Die bisher üblichen Sichtweisen - die wirkungsorientierte, die ursachenorientierte und die probabilistische Sichtweise - sind zu eng formuliert, weil sie die Gründe für die Nicht-Normalverteilung von Renditen und damit auch manche Risiken nicht erklären können (s. auch Armonat 2008, S. 157). Die Vorschläge aus der verhaltenswissenschaftlichen Literatur und aus diesem Artikel können einen Impuls zur Erweiterung der Risikomessung liefern. Insbesondere die Integration eines subjektiven Planungsansatzes eröffnet die Möglichkeit, Erkenntnisse aus der kognitiven Psychologie zu integrieren, die die Einschätzung von Praktikern - auch mittels Software - unterstützen könnten (Karelaia und Hogarth 2008; Lawrence et al. 2006). Der Fokus von Entscheidungsunterstützungssystemen sollte hierfür auf den Nutzer und die Effektivität des Systems gelenkt werden. Ein 
wichtiger Punkt, der in der bisherigen Literatur kaum behandelt wurde und neue Erkenntnisse erwarten lässt.

Interessenkonflikt P. Krieger, C. Lausberg und K. Wellner geben an, dass kein Interessenkonflikt besteht.

Open Access Dieser Artikel wird unter der Creative Commons Namensnennung 4.0 International Lizenz (http://creativecommons.org/licenses/by/4.0/deed.de) veröffentlicht, welche die Nutzung, Vervielfältigung, Bearbeitung, Verbreitung und Wiedergabe in jeglichem Medium und Format erlaubt, sofern Sie den/die ursprünglichen Autor(en) und die Quelle ordnungsgemäß nennen, einen Link zur Creative Commons Lizenz beifügen und angeben, ob Änderungen vorgenommen wurden. 


\section{Appendix}

Tab. 5 Beschreibung des Datensatzes zum Portfolio C3

\begin{tabular}{|c|c|c|c|}
\hline $\begin{array}{l}L f d . \\
N r\end{array}$ & Attributname & $\begin{array}{l}\text { Orig. } \\
\text { Daten }\end{array}$ & Beschreibung \\
\hline 1.0 & \multicolumn{3}{|l|}{ Basisangaben } \\
\hline 1.1 & Stichtag & JA & $\begin{array}{l}\text { Dieses Feld gibt den Monat sowie das Jahr des jeweiligen Tupels } \\
\text { an. Für jede Wohnung wurde zum Monatsanfang eine Moment- } \\
\text { aufnahme über die folgenden Attribute zur Verfügung gestellt. } \\
\text { Der Zeitraum des Datensatzes erstreckt sich von Januar } 2011 \text { bis } \\
\text { September } 2014 \text {. }\end{array}$ \\
\hline 1.2 & Nutzungsart & JA & $\begin{array}{l}\text { Dieses Feld gibt die Nutzungsart der vermietbaren Fläche an. } \\
\text { Für den Datensatz existieren ausschließlich Einträge mit der } \\
\text { Nutzungsart „Wohnraum“, die auch die Hauptnutzungsart der } \\
\text { jeweiligen Immobilie bildet. }\end{array}$ \\
\hline 2.0 & \multicolumn{3}{|c|}{ Identifikation der Wohnungseinheit } \\
\hline 2.1 & Objekt-ID & JA & $\begin{array}{l}\text { Eine eindeutige Nummer, die dem jeweiligen Wohnhaus durch } \\
\text { den Datensatz-Provider zugewiesen wurde. }\end{array}$ \\
\hline 2.2 & $\begin{array}{l}\text { Wohnungs- } \\
\text { ID }\end{array}$ & JA & $\begin{array}{l}\text { Eine eindeutige Nummer, die der jeweiligen Wohnung durch den } \\
\text { Datensatz-Provider zugewiesen wurde. }\end{array}$ \\
\hline 2.3 & $\begin{array}{l}\text { Vertragsnum- } \\
\text { mer }\end{array}$ & JA & $\begin{array}{l}\text { Eine eindeutige Nummer, die dem jeweiligen Mietvertrag durch } \\
\text { den Datensatz-Provider zugewiesen wurde. }\end{array}$ \\
\hline
\end{tabular}

3.0 Teilangaben zum Mietvertrag

3.1 Anzahl JA Dieses Feld gibt die Anzahl der Räume innerhalb der Wohnung Räume an. Die Angabe erfolgt in ganzen und halben Räumen, wobei sich die Definition noch nach der veralteten DIN 283 richtet.

3.2 Vereinbarte JA

Dieses Feld gibt die im Mietvertrag vereinbarte Mietfläche an. Mietfläche Die Angabe kann von der tatsächlichen Bruttogrundfläche der jeweiligen Wohnung abweichen. Inwieweit eine Differenz zur tatsächlichen Bruttogrundfläche besteht, kann anhand des Datensatzes nicht beurteilt werden. Der Datensatz-Provider hat hierzu keine weiteren Angaben zur Verfügung gestellt.

3.3 Soll-Miete JA

Dieses Feld gibt die aktuelle vereinbarte Soll-Miete an, die zum [€/mtl.] 3. Werktag des Stichtags fällig wurde. Dieses Feld enthält keine Angaben zu Mietminderungen, Mietrückständen und anderen Zahlungen, die die Soll-Miete betreffen können.

3.4 Soll-Miete NEIN; Dieses Feld gibt die Soll-Miete pro Quadratmeter an, die zum je$\left[€ / \mathrm{m}^{2} \mathrm{mtl}\right.$.] berechnet weiligen Stichtag fällig wurde. Dieses Feld wurde vom DatensatzProvider nicht geliefert, sondern aus der Soll-Miete geteilt durch die vereinbarte Mietfläche errechnet.

3.5 Mietvertrags- JA

Das vertraglich vereinbarte Startdatum des Mietvertrags.

beginn

3.6 Mietvertrags- JA ende

Das vertraglich festgehaltene Enddatum des Mietvertrags, welches durch die Kündigung des Mietvertrags festgehalten wurde. Für die Kündigung kommen alle vertraglich vereinbarten und rechtlich vorgeschriebenen Möglichkeiten in Betracht. Bei noch laufenden Mietverträgen wurde die Angabe „NULL“ hinterlegt. 


\section{Literatur}

Agarwal S, Ambrose BW, Huang H, Yildirim Y (2011) The term structure of lease rates with endogenous default triggers and tenant capital structure: theory and evidence. J Financial Quant Analysis 46(2):553-584

Ambrose BW, Ancel E, Griffiths MD (1992) The Fractal structure of real estate investment trust returns: the search for evidence of market segmentation and nonlinear dependency. Real Estate Econ 20(1):25-54

Andrews DWK, Guggenberger P (2003) A bias-reduced log-Periodogram regression estimator for the longmemory parameter. Econometrica 71(2):675-712

Armonat S (2008) Immobilienrenditen in finanzwirtschaftlichen Modellen: investmentorientierte Portfolio-Steuerung von Immobilienanlagen. Schriften des Forschungscenters Betriebliche Immobilienwirtschaft, Bd. 4. Rudolf Müller, Köln

Assaf A (2015) Long memory and level shifts in REITs returns and volatility. Int Rev Financial Analysis 42:172-182

Bachelier L (1900) Théorie de la spéculation. Gauthier-Villars, Paris

Baillie RT, Kapetanios G (2007) Testing for neglected Nonlinearity in long-memory models. J Bus Econ Stat 25(4):447-461

Baillie RT, Bollerslev T, Mikkelsen HO (1996) Fractionally integrated generalized autoregressive conditional heteroskedasticity. J Econom 74(1):3-30

Bals W, Wellner K (2016) Immobilien-Portfolio-Management und Immobilien-Asset-Management. In: Schäfer J, Conzen G (Hrsg) Praxishandbuch Immobilien-Investition, 3. Aufl. C.H. Beck, München, S 605-626

Barnett WA, Serletis A (2000) Martingales, nonlinearity, and chaos. J Econ Dyn Control 24(5-7):703-724

Baumeister A (2004) Risikomanagement bei Immobilieninvestments: Entscheidungshilfen für institutionelle Anleger, 1. Aufl. Deutscher Universitätsverlag, Wiesbaden

Belaire-French J, Opong KK (2013) A time series analysis of UK construction and real estate indices. J Real Estate Finance Econ 46(3):516-542

Benartzi S, Thaler RH (2001) Naive diversification strategies in defined contribution saving plans. Am Econ Rev 91(1):79-98

Blaikie NWH (1991) A critique of the use of triangulation in social research. Qual Quant 25(2):115-136

Bollerslev T (1987) A conditionally Heteroskedastic time series model for speculative prices and rates of return. Rev Econ Stat 69(3):542-547

Box GEP, Cox DR (1964) An analysis of transformations. J Royal Stat Soc Ser B 26(2):211-252

Broock WA, Scheinkman JA, Dechert WD, LeBaron B (1996) A test for independence based on the correlation dimension. Econom Rev 15(3):197-235

Brown GR, Matysiak GA (2000) Real estate investment: a capital market approach, 1. Aufl. Prentice Hall, London

Callahan M (2002) To hedge or not to hedge ...that is the question: empirical evidence from the north American gold mining industry 1996-2000. Finance Mark Inst Instr 11(4):271-288

Chinloy P, Cho M, Megbolugbe IF (1997) Appraisals, transaction incentives, and smoothing. J Real Estate Finance Econ 14(1-2):89-111

Cont R (2001) Empirical properties of asset returns: stylized facts and statistical issues. Quant Finance $1: 223-236$

Cont R, Tankov P (2004) Financial modelling with jump processes, 1. Aufl. Chapman \& Hall/CRC, Boca Raton

Cristelli M, Alfi V, Pietronero L, Zaccaria A (2010) Liquidity crisis, granularity of the order book and price fluctuations. Eur Phys J B 73(1):41-49

Crosby N, Hughes C (2011) The basis of valuations for secured commercial property lending in the UK. J Eur Real Estate Res 4(3):225-242

Croskerry P, Singhal G, Mamede S (2013) Cognitive debiasing 1: origins of bias and theory of debiasing. Bmj Qual Saf 22:ii58-ii64

DeBondt WFM, Thaler RH (1986) Further evidence on investor overreaction and stock market seasonality. J Finance 42(3):557-581

DeMiguel V, Garlappi L, Uppal R (2009) Optimal versus naive diversification: how inefficient is the $1 / \mathrm{N}$ portfolio strategy? Rev Financ Stud 22(5):1915-1953

Denzin NK (1970) The research act: a theoretical introduction to sociological methods, 1. Aufl. Aldine Publishers, Chicago

Diaz J III, Wolverton ML (1998) A longitudinal examination of the appraisal smoothing hypothesis. Real Estate Econ 26(2):349-358 
Diebold FX, Inoue A (2001) Long memory and regime switching. J Econom 105(1):131-159

Dornbusch R, Fischer S, Startz R, Schittko UK (2003) Makroökonomik, Internationale Standardlehrbücher der Wirtschafts- und Sozialwissenschaften, 8. Aufl. Oldenbourg, München

Elder J, Villupuram S (2012) Persistence in the return and volatility of home price indices. Appl Financial Econ 22(22):1855-1868

Eliazar II, Sokolov IM (2010) Measuring statistical heterogeneity: The Pietra index. Phys A: Stat Mech Appl 389(1):117-125

Fama EF (1965) The behavior of stock-market prices. J Bus 38(1):34-105

Fama EF (1970) Efficient capital markets: a review of theory and empirical work. J Finance 25(2):383-417

Flick U (1992) Triangulation revisited: strategy of validation or alternative? J Theory Soc Behav 22(2):175-197

Fox R, Taqqu MS (1986) Large-sample properties of parameter estimates for strongly dependent stationary Gaussian time series. Ann Stat 14(2):517-532

French N (2001) Decision theory and real estate investment: an analysis of the decision-making processes of real estate investment fund managers. MDE Manage Decis Econ 22(7):399-410

Gallagher M, Wood AP (1999) Fear of Overbuilding in the office sector: how real is the risk and Can we predict it? J Real Estate Res 17(1-2):3-32

GdW (2016) Wohnungswirtschaftliche Daten und Trends 2016/2017. Haufe-Lexware, Freiburg

Geweke J, Porter-Hudak S (1983) The estimation and Applicaiton of long memory time series models. J Time Ser Analysis 4(4):221-238

Gigerenzer G (2013) Risiko: Wie man die richtigen Entscheidungen trifft, 4. Aufl. btb, München

Giraitis L, Kokoszka P, Leipus R, Teyssière G (2003) Rescaled variance and related tests for long memory in volatility and levels. J Econom 112(2):265-294

Granger CWJ, Hyung N (2004) Occasional structural breaks and long memory with an application to the S\&P 500 absolute stock returns. J Empir Finance 11(3):399-421

Granger CWJ, Teräsvirta T (1999) A simple nonlinear time series model with misleading linear properties. Econ Lett 62(2):161-165

Grassberger P, Procaccia I (1983) Measuring the strangeness of strange attractors. Phys D: Nonlinear Phenom 9(1-2):189-208

Grenadier SR (1995) The persistence of real estate cycles. J Real Estate Finance Econ 10(2):95-119

Hamilton M, Lim LC, McCluskey W (2006) The changing pattern of commercial lease terms: Evidence from Birmingham, London, Manchester and Belfast. Prop Manag 24(1):31-46

Hammersley M (2008) Troubles with triangulation. In: Bergman MM (Hrsg) Advances in mixed methods research. SAGE, London, S 22-36

Hansainvest (2011) Jahresbericht zum 31. Dezember 2010: HANSAimmobilia. https://www.hansainvest. com/workspace/dokumente/lang_1/jahresbericht/HANSAimmobilia\%20Jahresbericht\%202010.pdf. Zugegriffen: 6. Apr. 2019

Hansz JA, Diaz J III (2001) Valuation bias in commercial appraisal: a transaction price feedback experiment. Real Estate Econ 29(4):553-565

Hendershott PH (2002) A comparison of upward-only and turnover leases. J Prop Invest Finance 20(6):513-524

Henger R, Voigtländer M (2015) Vereint in regionalen Unterschieden: Der deutsche Wohnungsmarkt 25 Jahre nach der der Wiedervereinigung. In: Institut der deutschen Wirtschaft Köln (Hrsg) IW Policy Paper Nr. 31. Institut der deutschen Wirtschaft Köln, Köln, S 1-23

Hirschman AO (1964) The Paternity of an Index. Am Econ Rev 54(5):761

Huschens S, Stahl G (2004) Granularität dominiert Korrelation. RISKNEWS 6:28-29

Interviewpartner 1 (2016), Risikowahrnehmung und Maßnahmen in der Praxis, Versicherung, persönliches Interview, München

Interviewpartner 2 (2016), Risikowahrnehmung und Maßnahmen in der Praxis, Assetmanagement, persönliches Interview, München

Interviewpartner 3 (2016), Risikowahrnehmung und Maßnahmen in der Praxis, Immobilienbewertung, telefonisches Interview

Jensen MJ (1999) Using wavelets to obtain a consistent OLS estimator of the long-memory parameter. J Forecast 18(1):17-32

Jirasakuldech B, Emekter R (2012) Nonlinear dynamics and chaos behaviors in the REIT industry: a preand post-1993 comparison. J Real Estate Portfolio Manag 18(1):57-77

Kahneman D (2016) Schnelles Denken, langsames Denken. Penguin, München

Kallberg JG, Liu CH, Pasquariello P (2002) Regime shifts in asian equity and real estate markets. Real Estate Econ 30(2):263-291 
Karelaia N, Hogarth RM (2008) Determinants of linear judgment: a Meta-analysis of lens model studies. Psychol Bull 134(3):404-426

Krystalogianni A, Tsolacos S (2004) Regime switching in yield structures and real estate investment. J Prop Res 21(4):279-299

Kurzrock B-M, Rottke NB, Schiereck D (2009) Factors that influence the performance of office properties. J Real Estate Portfolio Manag 15(1):59-73

Lausberg C (2001) Das Immobilienmarktrisiko deutscher Banken, 1. Aufl. Verl. Wissenschaft und Praxis, Sternenfels

Lawrence M, Goodwin P, O’Connor M, Önkal D (2006) Judgmental forecasting: A review of progress over the last 25 years. Int J Forecast 22(3):493-518

Ledergerber R (2012) Fragen und Antworten rund um Immobilien: Produkte und Hypotheken. In: UBS AG (Hrsg) UBS real estate focus: Immobilienmarkt Schweiz 2012. UBS AG, Zürich, S 39

Ledoit O, Wolf M (2003) Improved estimation of the covariance matrix of stock returns with an application to portfolio selection. J Empir Finance 10(5):603-621

Lee S (2006) The stability of the co-movements between real estate returns in the UK. J Prop Invest Finance 24(5):434-442

Li J, Li G, Zhou Y (2015) Do securitized real estate markets jump? International evidence. Pacific-basin Finance J 31:13-35

Lieser K, Groh AP (2014) The determinants of international commercial real estate investment. J Real Estate Finance Econ 48(4):611-659

Liow KH (2007) Cycles and common cycles in real estate markets. Int J Manag Finance 3(3):287-305

Liow KH (2009) Long-term memory in volatility: some evidence from international Securitized real estate markets. J Real Estate Finance Econ 39(4):415-438

Liow KH, Webb JR (2008) Nonlinear return dependence in major real estate markets. J Prop Res 25(4):285-319

Liow KH, Zhu H (2007) Regime switching and asset allocation: Evidence from international real estate security markets. J Prop Invest Finance 25(3):274-288

Liow KH, Zhu H, Ho DK, Addae-Dapaah K (2005) Regime changes in international Securitized property markets. J Real Estate Portfolio Manag 11(2):147-165

Lizieri C, Satchell SE (1997) Property company performance and real interest rates: a regime-switching approach. J Prop Res 14(2):85-97

Lizieri C, Ward C (2001) The distribution of commercial real estate returns. In: Knight J, Satchell SE (Hrsg) Return Distributions in Finance, 1. Aufl. Butterworth-Heinemann, Oxford, S 47-74

Lizieri C, Satchell SE, Wongwachara W (2012) Unsmoothing real estate returns: a regime-switching approach. Real Estate Econ 40(4):772-804

Lo AW (1991) Long-term memory in stock market prices. Econometrica 59(5):1279-1313

Luhmann N (1997) Die Moral des Risikos und das Risiko der Moral. In: Bechmann G (Hrsg) Risiko und Gesellschaft: Grundlagen und Ergebnisse interdisziplinärer Risikoforschung, 2. Aufl. VS, Wiesbaden, S $327-338$

Maitland-Smith JK, Brooks C (1999) Threshold autoregressive and Markov switching models: an application to commercial real estate. J Prop Res 16(1):1-19

Mandelbrot B (1960) The Pareto-Lévy law and the distribution of income. Int Econ Rev (Philadelphia) 1(2):79-106

Mandelbrot B (1963) The variation of certain speculative prices. J Bus 36(4):394-419

Markowitz H (1952) Portfolio selection. J Finance 7(1):77-91

McFee G (1992) Triangulation in research: two confusions. Educ Res 34(3):215-219

Merton RC (1976) Option pricing when underlying stock returns are discontinuous. J Financ Econ 3(1-2):125-144

Mikosch T, Starica C (2005) Changes of structure in financial time series and the GARCH model. Revstat Stat J 2(1):41-73

Müller M, Lausberg C, Lee S (2014) Is volatility the appropriate risk measure for direct real estate. Working paper, Bd. 3, S 1-21

Myer FCN, Webb JR (1993) Return properties of equity REITs, common stocks, and commercial real estate: a comparison. J Real Estate Res 8(1):87-106

Myer FCN, Webb JR (1994) Statistical properties of returns: Financial assets versus commercial real estate. J Real Estate Finance Econ 8(3):267-282

von Neumann J, Morgenstern O (1944) Theory of games and economic behavior, 1. Aufl. Princeton University Press, Princton 
Newell G, Peat M, Stevenson M (1996) Testing for evidence of nonlinear structure in Australian real estate market returns. Working Paper, Bd. 61, S 1-33

Norden L, Roosenboom P, Wang T (2016) The effects of corporate bond granularity. J Bank Finance 63:25-34

Northcraft GB, Neale MA (1987) Experts, amateurs, and real estate: an anchoring-and-adjustment perspective on property pricing decisions. Organ Behav Hum Decis Process 39:84-97

Nychka D, Ellner S, McCaffrey D, Gallant AR (1992) Finding chaos in noisy systems. J Royal Stat Soc Ser B 54(2):399-426

Paskelian OG, Hassan MK, Huff KW (2011) Are there bubbles in the REITs market? New evidence using regime-switching approach. Appl Financial Econ 21(19):1451-1461

Perron P, Qu Z (2010) Long-memory and level shifts in the volatility of stock market return indices. J Bus Econ Stat 28(2):275-290

Pfnür A, Armonat S (2001) Immobilienkapitalanlage institutioneller Investoren - Risikomanagement und Portfolioplanung. Arbeitspapiere des Arbeitsbereichs Öffentliche Wirtschaft am Fachbereich Wirtschaftswissenschaften der Universität Hamburg, Bd. 26. Universität Hamburg, Hamburg, S 1-97

Plantz F (2012) Grundlagen des Immobilien-Risikomanagements - Risikodarstellung und Methodenuntersuchung. https://ccpmre.de/wp-content/uploads/ftp-uploads/fachbuecher/CCPMRE_DP_2012_ Risiko.pdf. Zugegriffen: 6. Apr. 2019

Pástor L, Stambaugh RF (2003) Liquidity risk and expected stock returns. J Polit Econ 111(3):642-685

Qu Z, Perron P (2013) A stochastic volatility model with random level shifts and its applications to S\&P 500 and NASDAQ return indices. Econom J 16(3):309-339

Raydén T, Teräsvirta T, Asbrink S (1998) Stylized facts of daily return series and the hidden Markov model. J Appl Econom 13(3):217-244

Rea W, Oxley L, Reale M, Brown J (2013) Not all estimators are born equal: The empirical properties of some estimators of long memory. Math Comput Simul 93:29-42

Robinson PM (1995a) Gaussian Semiparametric estimation of long range dependence. Ann Stat 23(5): $1630-1661$

Robinson PM (1995b) Log-Periodogram regression of time series with long range dependence. Ann Stat 23(3):1048-1072

Rosenbluth G (1955) Measures of concentration. In: Universities-National Bureau (Hrsg) Business concentration and price policy, 1. Aufl. Princeton University Press, Princeton, S 57-99

Rottwilm C (2011) Immobilienanlage: Studenten als Renditebringer. http://www.manager-magazin.de/ finanzen/immobilien/a-792305.html. Zugegriffen: 6. Apr. 2019

Roy AD (1952) Safety first and the holding of assets. Econometrica 20(3):431-449

Ruelle D (1990) Deterministic chaos: the science and the fiction. Proc Royal Soc London Ser A 427(1873):241-248

Sandoval L Jr., Franca IDP (2012) “Correlation of financial markets in times of crisis”. Phys A Stat Mech Appl 391(1-2):187-208

Schierenbeck H, Lister M, Kirmße S (2008) Ertragsorientiertes Bankmanagement: Risiko-Controlling und integrierte Rendite-/Risikosteuerung, 9. Aufl. Bd. 2. Gabler, Wiesbaden

Schindler F (2009) Correlation structure of real estate markets over time. J Prop Invest Finance 27(6):579-592

Shiller RJ (1987) Investor behavior in the october 1987 stock market crash: survey evidence. Working Paper, Bd. 2446, S 1-41

Shimotsu K (2006) Simple (but effective) tests of long memory versus structural breaks. Working Paper, Bd. 1101, S 1-46

Shimotsu K (2010) Exact local whittle estimation of fractional integration with unknown mean and time trend. Econ Theory 26(2):501-540

Shintani M, Linton O (2004) Nonparametric neural network estimation of Lyapunov exponents and a direct test for chaos. J Econom 120(1):1-33

Slovic P (1987) Perception of risk. Science 236(4799):280-285

Slovic P (1999) Trust, emotion, sex, politics, and science: surveying the risk-assessment battlefield. Risk Analysis 19(4):689-701

Smith A (2005) Level shifts and the illusion of long memory in economic time series. J Bus Econ Stat 23(3):321-335

Smith SD, Woodward LR, Schulman CT (2000) The effect of the tax reform act of 1986 and Overbuilt markets on commercial office property values. J Real Estate Res 19(3):301-320

Smithson C, Simkins BJ (2005) Does risk management add value? A survey of the evidence. J Appl Corp Finance 17(3):8-17 
Strümpel B (1968) Die subjektive Liquidität als Zielvariable der ,Neuen Wirtschaftspolitik': Zum Funktionswandel der modernen Geldpolitik. In: Andeae CA, Hansmeyer KH, Scherhorn G (Hrsg) Geldtheorie und Geldpolitik: Günter Schmölders zum 65. Geburtstag, 1. Aufl. Duncker \& Humblot, Berlin, S 95-116

Swiss Finance \& Property AG (2015) Lebensplanung einer Immobilie. In: Swiss Finance \& Property AG (Hrsg) Quarterly Newsletter Juli 2015. Swiss Finance \& Property AG, Zug, S 7-8

Taleb NN (2007) The black swan: the impact of the highly improbable, 1. Aufl. Allen Lane, London

Teverovsky V, Taqqu MS, Willinger W (1999) A critical look at Lo's modified R/S statistic. J Stat Plan Inference 80(1-2):211-227

Vishwakarma VK, Paskelian OG (2012) Bubble in the Indian real estate market: identification using regime-switching methodology. Int J Bus Finance Res 6(3):27-40

Wellner K (2003) Entwicklung eines Immobilien-Portfolio-Management-Systems - Zur Optimierung von Rendite-Risiko-Profilen diversifizierter Immobilien-Portfolios, 1. Aufl. Books on Demand, Norderstedt

Wheaton WC (1987) The cyclic behavior of the national office market. Real Estate Econ 15(4):281-299

Wheaton WC, Torto RG, Sivitanides PS, Southard JA, Hopkins RE, Costello JM (2001) Real estate risk: a forward-looking approach. Real Estate Finance 18(3):20-28

Wilson PJ, Okunev J (1999) Long-term dependencies and long run non-periodic co-cycles: real estate and stock markets. J Real Estate Res 18(2):257-278

Wolf A, Swift JB, Swinney HL, Vastano JA (1985) Determining Lyapunov exponents from a time series. Phys D: Nonlinear Phenom 16(3):285-317

Wyman D, Seldin M, Worzala E (2011) A new paradigm for real estate valuation? J Prop Invest Finance 29(4-5):341-358

Yin RK (2014) Case study research: design and methods, 5. Aufl. SAGE, Los Angeles

Young MS (2008) Revisiting non-normal real estate return distributions by property type in the U.S. J Real Estate Finance Econ 36(2):233-248

Young MS, Graff RA (1995) Real estate is not normal: A fresh look at real estate return distributions. J Real Estate Finance Econ 10(3):225-259

Young MS, Lee S, Devaney SP (2006) Non-normal real estate return distributions by property type in the UK. J Prop Res 23(2):109-133

Zhou J (2011) Long memory in REIT volatility revisited: genuine or spurious, and self-similar? J Prop Res 28(3):213-232 